\title{
Differential Serotonergic Innervation of the Suprachiasmatic Nucleus and the Intergeniculate Leaflet and its Role in Circadian Rhythm Modulation
}

\author{
Elizabeth L. Meyer-Bernstein ${ }^{1}$ and Lawrence P. Morin ${ }^{1,2}$ \\ 1 Graduate Program in Neurobiology and Behavior and ${ }^{2}$ Department of Psychiatry and Behavioral Science, State \\ University of New York at Stony Brook, Stony Brook, New York 11794
}

Serotonergic innervation is believed to inhibit the effects of light on the mammalian circadian timing system. Two anatomical components of this system, the suprachiasmatic nucleus (SCN) and the intergeniculate leaflet (IGL), receive serotonergic input from midbrain raphe nuclei. The present studies use retrograde and anterograde tracing as well as neurotoxic lesion techniques to demonstrate that serotonergic cells in the median raphe nucleus (MR) project to the SCN and that serotonergic cells in the dorsal raphe nucleus (DR) project to the IGL.

Neurotoxic lesions were also used to investigate the effects of selective serotonin $(5-\mathrm{HT})$ neuron loss in the MR or DR on circadian rhythm parameters of animals entrained to a light/ dark cycle or housed in constant light. 5-HT depletion in the $\mathrm{MR}$, but not in the DR, induces an advance in onset, a delay in offset, and a longer duration of the nocturnal running-wheel

The hypothalamic suprachiasmatic nuclei (SCN) contain the mammalian circadian clock (Klein et al., 1991). Photic cues necessary for circadian rhythm entrainment are received by the retina and transmitted directly through the retinohypothalamic tract to the SCN (Stephan and Zucker, 1972; Moore, 1973; Pickard, 1982; Johnson et al., 1988a,b). Pholic information indirectly accesses the SCN through a retinal projection to the intergeniculate leaflet (IGL) of the lateral geniculate complex (Johnson et al., 1988b; Card and Moore, 1989; Harrington and Rusak, 1989; Zhang and Rusak, 1989; Takatsuji et al., 1991; Morin et al., 1992). The geniculohypothalamic tract, which connects the IGL to the SCN, is not necessary for entrainment but may modulate the effects of light on the circadian clock (Harrington and Rusak, 1986, 1988; Pickard et al., 1987).

Serotonin (5-HT) cells in the mesencephalic raphe complex give rise to the third major SCN input (Azmitia and Segal, 1978; Moore et al., 1978; van de Kar and Lorens, 1979) and also innervate the IGL (Mantyh and Kemp, 1983; Morin et al., 1992). Abundant evidence implicates 5-HT as an inhibitory transmitter that modulates the effects of light on circadian rhythmicity. Application of 5-HT or receptor agonists to the SCN block both light-induced phase shifts and FOS protein induction during the

\footnotetext{
Received July 27, 1995; revised Nov. 30, 1995; accepted Dec. I8, 1995.

This research was supported by National Institute of Neurological Disorders and Stroke Grant NS22168. We wish to thank Jane Blanchard for her superb technical assistance.

Correspondence should be addressed to Dr. Lawrence P. Morin, Department of Psychiatry and Behavioral Science, State University of New York at Stony Brook, Stony Brook, NY 11794-8101.

Copyright (C) 1996 Society for Neuroscience $0270-6474 / 96 / 162097-15 \$ 05.00 / 0$
}

activity phase. Circadian rhythm disruption in constant light is also more frequent in hamsters with MR lesions. A second experiment was designed to investigate the relationship between lesion location, 5-HT-immunoreactive (5-HT-IR) fiber loss, and behavioral changes. Destruction of $5-\mathrm{HT}$ neurons in the MR causes 5-HT-IR fiber loss in the SCN, which may account for the observed changes in circadian parameters. DR lesions result in 5-HT-IR fiber depletion of the $I G L$, with no associated changes in the entrained rhythm. The anatomical and behavioral results support the view that a $5-\mathrm{HT}$ projection from the MR mediates 5-HT effects on circadian rhythm regulation in hamsters.

Key words: serotonin; circadian rhythm; suprachiasmatic nucleus; intergeniculate leaflet; locomotor activity; raphe nucleus

subjective night (Shibata and Moore, 1988; Selim et al., 1993; Glass et al., 1994; Rea et al., 1994). During the subjective day, when endogenous 5-HT release is low (Ramirez et al., 1987; Glass et al., 1992, 1993; Poncet et al., 1993), these same pharmacological agents induce circadian rhythm phase advances (Prosser et al., 1990, 1992; Medanic and Gillette, 1992; Tominaga et al., 1992; Edgar et al., 1993; Prosser et al., 1993, 1994a) and decrease endogenous levels of SCN c-fos expression (Prosser et al., 1994b). In addition, electrophysiological data indicate that 5-HT or receptor agonists inhibit the excitatory effects of light in the SCN (Miller and Fuller, 1990; Ying and Rusak, 1994) and the LGN complex (Meijer and Groos, 1988; Ying et al., 1993). In the absence of 5-HT, entrained locomotor rhythmicity persists, but the nocturnal activity phase is expanded. Under constant light (LL) conditions, 5 HT depleted hamsters have longer circadian periods and a greater propensity toward rhythm "splitting" or more severe rhythm disruption (Smale et al., 1990; Morin and Blanchard, 1991b; Morin, 1992).

Intraventricular application of the transmitter-specific neurotoxin 5,7-dihydroxytryptamine (DHT) indiscriminately eliminates serotonergic innervation of the SCN and IGL (Smale et al., 1990; Morin and Blanchard, 1991b; Morin, 1992). This method induces extensive damage to the dorsal raphe nucleus (DR), without producing readily observable changes in the median raphe nucleus (MR) (Smale et al., 1990; Morin and Blanchard, 1991b; Morin, 1992). The implication has been that DR 5-HT cells regulate circadian rhythmicity. No study, however, has addressed directly whether the DR or the MR mediates the alterations in running rhythm parameters.

Anatomical tract tracing studies conducted in the rat are incon- 
sistent in their description of SCN and IGL/LGN innervation by raphe neurons (Conrad et al., 1974; Pierce et al., 1976; Azmitia and Segal, 1978; Moore et al., 1978; Vertes and Martin, 1988; Vertes, 1991; Sim and Joseph, 1993). In addition, little effort has been made to differentiate between projections of the DR and the MR. Lack of injection site specificity after tritiated amino acid placement in the raphe complex does not permit definite conclusions concerning the source of SCN or IGL innervation. Despite obvious differences in the data across studies, a general belief has been that the SCN receives its primary serotonergic innervation from the DR. This notion has been supported by studies using intrahypothalamic retrograde tracer application, which reveal labeled cells in the DR and to a lesser degree in the MR (Bina et al., 1993). Retrograde studies consistently demonstrate a DR projection to the LGN (Pasquier and Villar, 1982; Waterhouse et al., 1993), but the IGL has not been investigated specifically. In addition, no previous studies have demonstrated that individual DR or MR neurons projecting to the SCN or IGL are serotonergic.

The first goal of the present studies was to describe, using both retrograde and anterograde techniques, the origin of serotonergic projections to the SCN and IGL in the hamster. The second goal was to determine the contribution of dorsal and median raphe serotonergic systems to circadian locomotor rhythm regulation.

\section{ABBREVIATIONS}

$3 \mathrm{v} 3 \mathrm{rd}$ ventricle

$4 \mathrm{v} 4$ th ventricle

ATg anterior tegmental nucleus

aq cerebral aqueduct

cg central gray

$\mathrm{CLi}$ caudal linear raphe nucleus

ep cerebral peduncle

DR dorsal raphe nucleus

DLG dorsal lateral geniculate nucleus

DTg dorsal tegmental nucleus

IC inferior colliculus

IGL intergeniculate leaflet

IP interpeduncular nucleus

LPB lateral parabrachial nucleus

LTN lateral terminal nucleus

mlf medial longitudinal fasiculus

MG medial geniculate nucleus

MR median raphe nucleus

ot optic tract

ox optic chiasm

$\mathrm{PnO}$ pontine reticular nucleus oral

RN red nucleus

RtTg reticulotegmental nucleus pons

$\mathrm{SC}$ superior colliculus

SCN suprachiasmatic nucleus

SN substantia nigra

VLG ventral lateral geniculate nucleus

VTA ventral tegmental area

VTg ventral tegmental nucleus

xscp decussation of superior cerebral peduncle

ZI zona incerta

\section{MATERIALS AND METHODS}

Male Syrian hamsters (90-100 gm, Charles River/Lakeview, Wilmington, MA) were housed individually under a $14 \mathrm{hr}$ light/10 hr dark photoperiod (LD14:10) in polypropylenc cages $(45 \times 20 \times 20 \mathrm{~cm})$ with food and water available ad libitum. Animals were acclimated to laboratory conditions for a minimum of 3 weeks before participation in an experiment. Each hamster destined for a behavior study was transferred to a similar cage containing a running wheel $(17 \mathrm{~cm}$ diameter $)$ to which it had free access. Wheel revolutions were counted by computer in 5 min bins. Surgery was conducted stereotaxically on deeply anesthetized hamsters (sodium pentobarbital, $100 \mathrm{mg} / \mathrm{kg}$ ).

\section{Retrograde tracer application}

Cholera toxin B subunit (CTb) (List Biological, Campbell, CA), $1 \%$ in distilled water, or Fluoro-Gold (FG) (Fluorochrome, Englewood, CO), $4 \%$ in saline, was deposited iontophoretically in the SCN or IGL through a glass micropipette (20-40 $\mu \mathrm{m}$ tip diameter). A direct positive current $(1.5-2.0 \mu \mathrm{A})$ was passed through the micropipette using a Stoelting Precision Current Source alternating $7 \mathrm{sec}$ on/7 sec off for $5-15 \mathrm{~min}$. Fast blue (FB) (Sigma, St. Louis, MO) (0.02 $\mu \mathrm{l}, 3 \%$ in distilled water) was injected into the SCN with a Hamilton syringe. Unilateral injections to the SCN were made at a $10^{\circ}$ angle using the following coordinates: 0.6 $\mathrm{mm}$ anterior to bregma, $1.25 \mathrm{~mm}$ lateral to bregma, $7.7 \mathrm{~mm}$ ventral to the dura. CTb or FG was unilaterally applied to the IGL at the following coordinates: $1.8 \mathrm{~mm}$ posterior to bregma, $3.4 \mathrm{~mm}$ lateral to bregma, 4.4 $\mathrm{mm}$ ventral to the dura. The injection needle or electrode remained in place for 5-15 min postinjection to minimize spread during withdrawal. Animals survived 2-10 d postinjection.

\section{Anterograde tracer application}

Phaseolus valgaris leucoagglutinin (PHAL) (Vector Labs, Burlingame. $\mathrm{CA}), 2.5 \%$ in PBS, pH 8.0 , was iontophoretically applied through a glass micropipette (tip diameter $40-60 \mu \mathrm{m}$ ). The micropipette was positioned at a $20^{\circ}$ angle and aimed at either the median raphe $(4.5 \mathrm{~mm}$ posterior to bregma, $2.3 \mathrm{~mm}$ lateral to bregma, $6.5 \mathrm{~mm}$ ventral to the dura) or dorsal raphe $(4.5 \mathrm{~mm}$ posterior to bregma, $1.6 \mathrm{~mm}$ lateral to bregma, $4.8 \mathrm{~mm}$ ventral to the dura). A Stoclting Precision Current Source was used to pass $8 \mu \mathrm{A} \mathrm{DC}(7 \mathrm{sec}$ on $/ 7 \mathrm{sec}$ off $)$ for $20-30 \mathrm{~min}$ at either location. The micropipette remained in position 5-15 min postinjection to minimize spread during withdrawal. Survival time of $10 \mathrm{~d}$ was allowed for anterograde transport of the tracer.

\section{Neurotoxic lesions}

Thirty minutes before surgery, each animal received an intraperitoneal injection of desipramine hydrochloride (Sigma) ( $25 \mathrm{mg} / \mathrm{kg}$ in saline) to protect catecholaminergic neurons. Each experimental animal was anesthetized, placed in a stereotaxic apparatus, and given a single injection of the 5-HT-specific neurotoxin, DHT (Sigma), $14.4 \mu \mathrm{g}$ free base in $0.5 \mu \mathrm{l}$ vehicle $/ 0.5 \%$ ascorbic acid ( $/ \mathrm{v} 86 \%)$ combined with the retrograde tracer FG ( $4 \%$ in saline) ( $v / v$ 14\%). FG was used as an index of the DHT injection site in subscquent anatomical analysis. Control $(\mathrm{CON})$ animals received an equal volume of vehicle. Injections were made with a Hamilton syringe positioned at a $20^{\circ}$ angle and aimed at either the median (coordinates: $4.2 \mathrm{~mm}$ posterior to bregma, $2.3 \mathrm{~mm}$ lateral to bregma, 6.7 $\mathrm{mm}$ ventral to dura) or dorsal raphe (coordinates: $4.2 \mathrm{~mm}$ posterior to bregma, $1.7 \mathrm{~mm}$ lateral to bregma, $5.15 \mathrm{~mm}$ ventral to dura).

\section{Behavior experiments}

Experiment 1 . Experimental hamsters, stably entrained to LDI4:10, received DHT neurotoxic lesions of the DR (DR-DHT, $n=8$ ) or the MR (MR-DHT, $n=10)$. CON hamsters received similarly placed vehicle injections (MR-V, $n=5$; DR-V, $n=5$ ). Hamsters remained in LD 14:10 for 8 weeks postsurgery, at which time they were transferred to LL ( 100 lux) until termination of the experiment $(100 \mathrm{~d})$. Hamsters survived 95 additional days in LD 14:10.

Experiment 2. Destruction of the serotonergic system by intraventricular application of DHT is followed eventually by substantial fiber regeneration in both SCN and IGL (Bosler et al., 1992; Morin, 1992). Such regrowth was expected to decrease greatly the likelihood of correlating behavioral change with fiber loss in these two nuclei in the long-term animals of experiment 1 . Therefore, after experiment 1 established that local raphe application of DHT produced behavioral changes, experiment 2 was performed to study acutely the relationship between behavior and 5-HT system damage induced by DHT lesions of the MR $(n=15)$ or DR $(n=10)$. CON animals received vehicle injections (MR-V, $n=4$; DR-V, $n=3$ ). The procedures for this experiment were identical to those of experiment 1 , but survival time was only $7-10 \mathrm{~d}$ postsurgery. 


\section{Histological procedures}

All animals were deeply anesthetized with sodium pentobarbital and perfused transcardially with $0.9 \%$ saline followed by $300 \mathrm{ml}$ of $4 \%$ paraformaldehyde in $0.1 \mathrm{M}$ PBS. Brains were removed and postfixed overnight before cryoprotection in $30 \%$ sucrose. Four series of coronal sections $(40 \mu \mathrm{m})$ through the diencephalon and mesencephalon were cut with a freezing-stage microtome and collected into $0.01 \mathrm{M}$ PBS. Sections included the rostral serotonergic system cell groups, which ascend to provide 5-HT innervation to the diencephalon (Azmitia, 1978; Tork, 1990; Jacobs and Azmitia, 1992). The ascending serotonergic system is divisible into ventral and dorsal cell groups. Because the ventral cell groups, consisting of the midbrain and pontine MR as well as the paramedian raphe, are closely juxtaposed, these groups were not partitioned for this analysis. Similarly, the dorsal cell groups consist of both midbrain and pontine DR, which are analyzed here as the DR.

Immunohistochemistry. Immediately after cutting, one set of sections was pretreated for $5 \mathrm{~min}$ with $1 \% \mathrm{H}_{2} \mathrm{O}_{2}$. After they were rinsed, sections were incubated in the primary antibody diluent ( $1 \%$ bovine serum albumin, $3 \%$ Triton $\mathrm{X}-100,3 \%$ goat or rabbit serum in PBS) for $30 \mathrm{~min}$ and then transferred to the appropriate primary antibody solution for $2-4 \mathrm{~d}$ at $4^{\circ} \mathrm{C}$. The following polyclonal antibodies were used: rabbit anti-5-HT, 1:2000 (Eugenetech, Allendale, NJ); rabbit anti-FG, 1:2000) (Chemicon, Temecula, C.A); goat anti-C.Th, 1:25,000 (1 ist Biological); goat anti-PHA $(\mathrm{E}+\mathrm{L}), 1: 2000$ (Vector); rabbit anti-PHAL, 1:2000) (Dako, Carpinteria, CA). A monoclonal rat anti-5-HT antibody, 1:50 (Accurate, Westbury, NY), was also used. Sections were rinsed and incubated in a biotinylated secondary antibody (goat anti-rabbit or rabbit anti-goat, 1:20); Vector) for $90 \mathrm{~min}$ at room temperature. Subsequently, sections were rinsed and incubated for 90 min at room temperature in avidin-biotin complex (1:100) (Vector $A B C$ Elite kit). Visualization of the avidin-biotin complex was achieved with diaminobenzidine (DAB) as the chromogen. Osmium tetroxide or nickel ammonium was used for intensification of the reaction product.

A fluorescent double-label technique was performed on a second set of sections from brains injected with retrograde tracer to confirm neurotransmitter identity of labeled cells. The following modifications were made to the above protocol. Brains injected with FG were incubated in 5-HT primary antibody with subsequent incubation in a Texas redconjugated goat anti-rabbit secondary antibody. Brains injected with CTb were incubated in a primary antibody cocktail consisting of anti-5-HT and anti-CTb (1:5000). The fluorescent method was less sensitive than the DAB method for identifying retrogradely labeled cells, and the 5 -HT cells werc consistently intensely stained. Therefore, the concentration of antiCTb was increased in the double-label protocol to insure identification of both labels in individual cells. The sccondary incubation was with a Texas red-conjugated donkey anti-goat antibody (1:50) (Jackson ImmunoResearch, West Grove, PA) for visualization of retrogradely labeled cells and with a biotinylated donkey anti-rabbit antibody (1:500) (Jackson ImmunoResearch). A third incubation in fluorescein isothiocyanateconjugated avidin D (Vector, $3 \mu \mathrm{l} / \mathrm{ml}$ ) was used to label 5 -HT cells.

Injection site. An atlas of the hamster diencephalon and mesencephalon was constructed from a series of cresyl violet-stained sections. Retrograde tracer application sites in the IGL or SCN and PHAL injection sites in the midbrain were mapped onto the appropriate sections of this atlas. The extent of tracer spread was drawn on atlas maps using a drawing tube. The locations of DHT injections, as indicated by FG-IR, were mapped similarly.

Cell body and fiber distribution. Retrogradely labeled midbrain cells, from both single- and double-labeled series of sections, were mapped onto the standard atlas sections. A drawing tube was used in instances in which DAB was used as the chromogen. In each brain, retrogradely labeled cell counts were calculated from a single series of sections and thus reflect approximately one-quarter of the expected labeled cells in the entire midbrain.

All sections containing the SCN or IGL in brains processed for 5-HT-IR or PHAL-IR were evaluated visually under the microscope for fiber distribution and density.

5-HT cell counts. The 5-HT-IR cells remaining after DHT injection into the DR or MR were counted to provide an index of lesion extent. In experiment 1 , cell counts were made on stained sections in which the 5-HT polyclonal antibody was used. This antibody produced a high background reaction product that made cell counting difficult. Nevertheless, our intent was to acquire an index of lesion success, and the polyclonal antibody proved adequate for that task. Cell counts in experiment 2 were performed on tissue stained with a monoclonal antibody, which produced a much lighter background, and are more reliable becausc of a better signal-to-noise ratio. All 5-HT-IR cells in each brain were counted throughout the full rostrocaudal extent of both nuclei (eight sections for MR, nine sections for DR) in one series of sections using a microcomputer imaging device (MCID) (Imaging Research, Brock University, Ontario, Canada). Cell counts can be compared across groups with similar histological procedures and are not intended to be a precise count of cells in the raphe.

Densitometry. In the DHT-treated animals, the entire $\mathrm{SCN}$ from a single series of sections processed for 5-HT-IR using the polyclonal antibody was examined visually. The section with the densest 5-HT-IR in the SCN was selected for further analysis. The relative optical density (ROD) in a single SCN of each animal was calculated using the ROD function of the MCID imaging system.

\section{Behavioral Measures}

Activity phase onset, offset, and duration $(\alpha)$ were obtained for a period of 5-10 d pre- and posttreatment when the animals were entrained to LD 14:10. In both experiments, presurgical measures were obtained for the $10 \mathrm{~d}$ immediately before surgery. In experiment 1 , activity-phase parameters were cvaluated for both acutc (beginning $\sim 10 \mathrm{~d}$ postsurgery) and long-term effects (beginning $\sim 6$ wecks postsurgery). In experiment 2 , the acute behavioral effects of DHT were measured for 5-8 d beginning 2 d postsurgery. Activity onset is defined as the time point, relative to lights off, at which the wheel revolutions per $5 \mathrm{~min}$ bin first exeed $5 \%$ of the daily maximum number of wheel revolutions per bin (Smale et al., 1990). Activity offset is defined als the time point, relative to lights off, at which the 98 th percentile of daily activity is reached. This was empirically determined by pilot work to be the least variable indicator of activity offset. $\alpha$ is the difference between these two values.

Circadian period $(\tau)$ and $\alpha$ were measured during a $10 \mathrm{~d}$ period in $L L$. Running records often became less precise in LL, in particular the time of activity offset. Under these conditions, the 95th percentile was the least variable index of activity phase offset, and the definition of $\alpha$ in LL was modified to include the time interval between the 1st and 95th percentiles of total daily activity.

The activity of MR-DHT animals often was disrupted rapidly in LL. Therefore, the specific postsurgical interval during which rhythmic behavior was measured varied between animals. Activity data from all animals, however, were analyzed over a minimum of $5 \mathrm{~d}$ during days 10-30 of LL. The onset of rhythm disruption was defined as the day on which the activity phase began to deviate from the previously stable circadian locomotor rhythm into a pattern of locomotor activity that was not rhythmic solely in the circadian domain (Morin and Blanchard. 1991 b; Morin, 1993). The onset and degree of rhythm disruption were assessed visually by two independent observers.

\section{Statistics}

Treatment effects on behavior were initially evaluated with a one-way ANOVA followed by relevant planned comparisons. A repeated measures analysis was used in experiment 1 . Because of the extended duration of experiment 1, deaths occurred before its conclusion, thereby making it impossible to assess lesion placenent in two MR-DHT-, four DR-DHT-, and five vehicle-treated animals. Nevertheless, all animals were included in the behavioral analysis, and each was retained in its originally assigned group. Statistical comparisons between these groups were expected to be conservative because of the likelihood that some individuals would have incomplete or missed lesions. In both experiments, MR-V and DR-V groups did not differ on any behavioral measure and were combined into a single CON group for all subsequent analyses. Survival analysis was used to evaluate between-group differences in days to onset of noncircadian behavior in LL. This analysis is able to accommodate data from animals who do not survive $100 \mathrm{~d}$ in LL. Fisher's exact probability tests were also used to assess group differences in LL.

To determine the presence of anatomical differences, either a Student's two-tailed $t$ test or a one-way ANOVA was performed when appropriate. Necessary between-group comparisons were made with Neuman-Keuls post-hoc tests in experiment 1 . In experiment 2, planned between-group comparisons were made. Correlation analysis was used to cvaluate the relationship between behavioral and anatomical data.

Results are summarized by a mean \pm SE. Statistical values from the comparisons are presented unless noted otherwise. 


\begin{tabular}{lllllll}
\hline \multicolumn{6}{l}{ Table 1. Distribution of midbrain cells projecting to the SCN } \\
Case:tracer & DR & MR & VTg & DTg & CG & LPB \\
\cline { 2 - 7 } 17:FB & 0 & 5 & 2 & 0 & 0 & 0 \\
15:FG & 0 & 10 & 2 & 0 & 0 & 0 \\
$43: \mathrm{CTb}$ & 0 & 6 & 0 & 0 & 0 & 0 \\
$11: \mathrm{CTb}^{2}$ & 0 & $10(5)^{a}$ & 1 & 3 & 0 & 0 \\
14:CTb & 0 & $20(11)$ & 0 & 3 & 4 & 0 \\
$8: \mathrm{CTb}^{b}$ & $1(1)$ & $13(6)$ & 0 & 0 & 12 & 9 \\
\hline
\end{tabular}

"Cells immunopositive for serotonin indicated in parentheses.

${ }^{b}$ Tracer spread into surrounding hypothalamus.

\section{RESULTS}

\section{Neuroanatomy}

Retrograde analysis: raphe projections to the $S C N$

The median raphe was the primary site of retrogradely labeled cells in the midbrain after SCN injections (Table 1, Fig. 1). Each brain with a successful SCN injection site had approximately the same distribution of cells scattered through the full rostrocaudal extent of the MR (Fig. 1). Specific clusters of retrogradely labeled cells within the MR were not evident, but cells were encountered more often in the caudal sections of the nucleus. With the exception of one cell (case 8), the DR and caudal linear raphe (CLi) were devoid of any retrogradely labeled neurons after SCN injections.

Injections of either $\mathrm{FG}, \mathrm{FB}$, or CTb in the $\mathrm{SCN}(n=6)$ generally had minimal spread beyond SCN boundaries (Figs. 2, 3), and the number of cells located outside the MR was related to the degree of spread outside the SCN (Table 1). In cases 11, 15, 17, and 43 , the injection site was limited to the SCN, and labeled cells were found almost exclusively in the MR. These injections labeled an average of 9.75 cells in the eight midbrain sections examined, $79 \%$ of which were found in the MR. There are an estimated 43 $\pm 9(n=6) \mathrm{MR}$ cells afferent to the SCN per brain. Cells labeled outside the MR were found consistently in the ventral tegmentum, and in case 11 the dorsal tegmentum contained cells as well. Slightly larger injection sites (cases 8 and 14) labeled many more cclls in the midbrain (mean $=31$ ), 53\% of which were found in the MR. In both cases, labeled cells were also found in the central gray, and the largest injection (case 8) labeled cells in the lateral parabrachial nucleus and a single DR cell.

Analysis of retrogradely labeled cells colocalizing 5-HT-IR (cases 8,11, and 14) shows that the median raphe is the only locus of serotonergic cells projecting to the SCN (Table 1, Fig. 1). Approximalely $50 \%$ of retrogradely labeled cells in the MR also contain 5-HT-IR. Equal proportions of retrogradely labeled 5-HT-IR cells exist across all sections of the median raphe. Similarly identifiable cells are seldom found outside the MR. The only exception was the single cell in the DR of case 8 that had extensive label spread into the hypothalamus dorsal to the SCN.

\section{Retrograde analysis: raphe projections to the IGL}

Retrograde tracer injected into the IGL labeled numerous cells throughout the DR and other midbrain regions (Table 2, Fig. 1). In addition to the dorsal raphe, the most consistent midbrain sites with labeled cells were the superior colliculus, dorsal tegmentum, and caudal parabrachial regions. None of these regions contain 5-HT-IR cells.

Successful injection sites $(n=4)$ included the IGL and adjacent portions of the lateral geniculate and zona incerta (Fig. 4). All animals had similar distributions of cells throughout the DR. Cells labeled in the DR were numerous in the rostral two-thirds of the nucleus and sparse in the caudal DR. An estimated $47 \pm 10(n=$ 4) dorsal raphe cells project to the IGL. Rarely was a labeled cell found in the MR, and none were found in the CLi.

Approximately $38 \%$ of the labeled cells found in the DR were 5-HT-IR (Table 2, Fig. 1). The only retrogradely labeled 5-HT-IR non-DR cell was found in the MR.

\section{Anterograde analysis: MR projections to the SCN and IGL}

Eight animals had successful injections of PHAL into the MR. In each case, PHAL-IR fibers were found in the SCN, with few or none found in the IGL (Table 3).

In four cases, injections labeled a substantial fiber plexus in the SCN. In two cases (1 and 14), fibers were concentrated in the medial and ventral regions of the nucleus, closely resembling the normal 5-HT fiber distribution in the SCN (Fig. 5A). Cases 15 and 22 had more ubiquitous fibers in the SCN. Three injection sites (cases 1, 15, and 22) that resulted in dense innervation of the SCN included the entire MR. The injection site in case 14 was similar, but no PHAL-IR cells were found in the rostral MR (Fig. 6). This was the smallest injection site and was limited to the MR complex. The injection sites of cases 1, 15, and 22 were centered in the MR, with spread extending slightly rostral to include portions of the interpeduncular nucleus and, in the caudal midbrain, the ventral tegmental nucleus. Of the four animals with numerous PHAL-IR fibers in the SCN, no injection site had PHAL spread into the DR, except in case 22 in which PHAL spread into the ventral region of the caudal DR and the dorsal tegmental nucleus.

Four additional animals also had successful injections of PHAL in the MR, but only a few labeled fibers were found in the SCN of each case. Label spread did not incorporate the entire MR and may not have labeled sufficient numbers of SCN afferent cells to provide a robust PHAL-IR fiber plexus in the SCN.

The number of labeled fibers in the SCN was not related to the rostrocaudal placement of the PHAL injection site. The degree of spread into adjacent nonraphe areas was similarly unrelated to PHAL-IR fiber density in the SCN.

In three cases with PHAL injections into the MR, no labeled fibers could be found in the IGL. In the remaining five animals, a few fibers were found in the more medial part of the ventral lateral geniculate (VLG) and the IGL (Fig. $5 B$ ).

\section{Anterograde analysis: DR projections to the SCN and IGL}

Seven animals had successful injections of PHAL in the DR. In all cases, PHAL-IR fibers were found in the IGL. With one exception (case 25), none had significant numbers of labeled fibcrs in the SCN (Table 3).

In five cases $(6,7,12,24$, and 29$)$, tracer spread excluded the MR, and the SCN of each essentially was devoid of fibers (Fig. $5 C$ ). In case 23, PHAL spread into the dorsal tip of the MR but did not label any fibers in the SCN. Only case 25 had substantial

Table 2. Distribution of midbrain cells projecting to the IGL

\begin{tabular}{lcllllrr} 
Case:tracer & DR & MR & VTg & DTg & CG & SC & LPB \\
\hline $14: \mathrm{FG}$ & $7(2)^{a}$ & $1(1)$ & 0 & 0 & 0 & 22 & 3 \\
$46: \mathrm{CTb}$ & $19(9)$ & 0 & 0 & 2 & 0 & 109 & 10 \\
$31: \mathrm{CTb}$ & $11(3)$ & 3 & 0 & 2 & 0 & 78 & 0 \\
$39: \mathrm{CTb}$ & $10(4)$ & 0 & 0 & 2 & 1 & 30 & 0 \\
\hline
\end{tabular}

"Cells immunopositive for serotonin indicated in parentheses. 

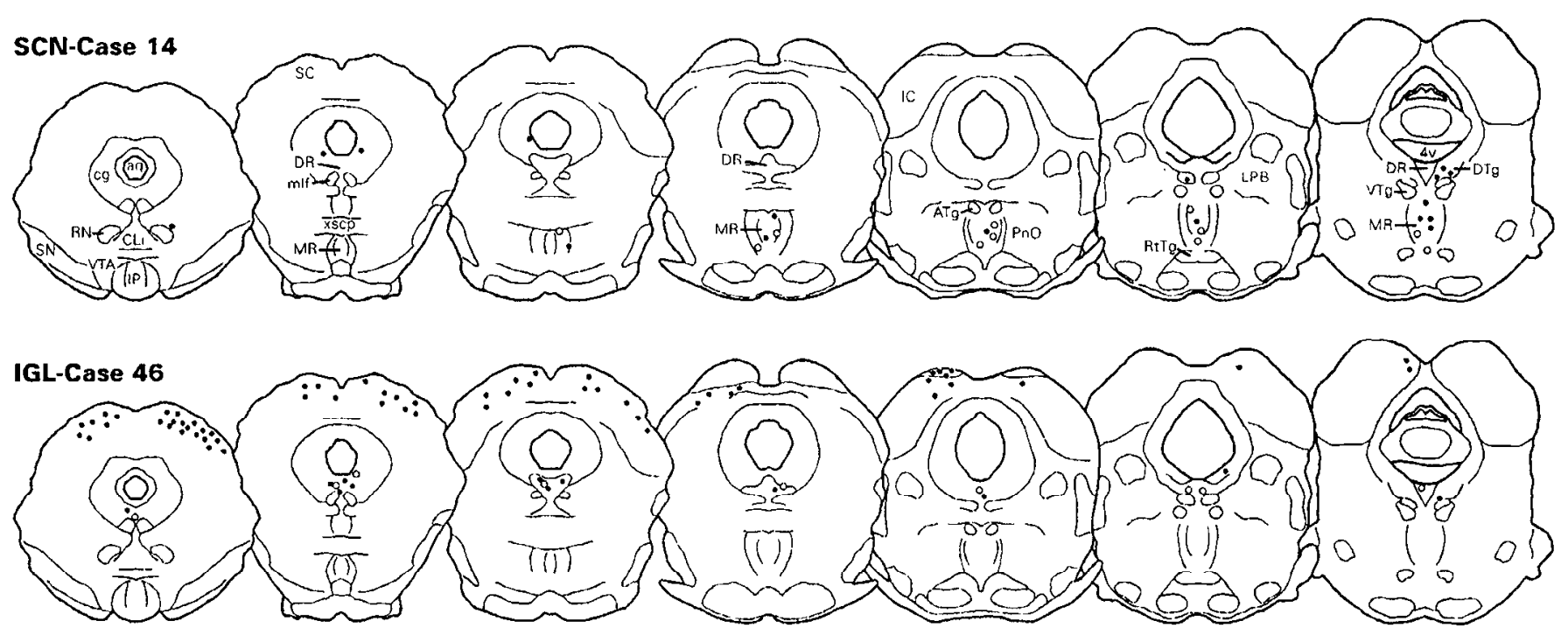

Figure 1. Distribution of single- (closed circles) and double-labeled cells (open circles) throughout the rostrocaudal extent of the midbrain after retrograde tracer injection into the SCN (top) or the IGL (botom). Single-labeled cells are (TT)-IR. Double-labeled cells are immunoreactive for both CTh and 5-HT. For definitions of abbreviations in all figures, see list located before Materials and Methods.

label spread into the dorsal MR and PHAL-IR fibers in the SCN. In the ventral hypothalamus, the absence of PHAL-IR fibers was specific to the SCN. PHAL-IR fibers were found in the surrounding hypothalamus and the retrochiasmatic area and coursing through the optic chiasm.

In the IGL, two distinctly different patterns of PHAL-IR fibers were observed, depending on the rostrocaudal placement of the tracer. In cases 7 and 24, the injection sites were centered in the mid-DR, and label spread the full rostrocaudal extent of the DR (Fig. 6). The numcrous PHAL fibers that were found were distributed throughout the IGL and VLG of these brains and resembled the normal 5-HT-IR distribution (case 7, Fig. 5D). In some sections, fibers were densest in the lateral half of the IGL and VLG. In case 24, label-spread extended into the rostral DR only on the right side, but became distributed more bilaterally in the mid- to caudal DR, resulting in a mixed fiber distribution in the geniculate. A medial fiber group was clearly labeled (Fig. 5E), but fibers in the lateral VLG and IGL were present only ipsilateral to the side of the spread.

The PHAL injection site was centered in the mid- to caudal DR in four cases $(6,12,23$, and 25), and PHAL-IR fibers were found consistently in the medial half of the VLG and IGL. PHAL fibers exiting the DR ventrolaterally reach the lateral edge of the midbrain, at which point they ascend to more rostral brain regions. At the level of the LGN, fibers turn dorsally and course through the medial VLG and vIGL to enter the IGL from a ventromedial location.

\section{Behavioral experiment 1}

\section{Histology}

DHT application to the DR significantly reduced 5-HT-IR cells in the DR group compared with the CON group (Fig. 7) $(t=3.78$; $p<0.01)$. Similarly, neurotoxin application to the MR caused a significant reduction of 5-HT-IR neurons in the MR group compared with the CON group (Fig. 7) $(t=4.04 ; p=0.002)$.

Despite the length of this experiment and the likelihood of fiber regrowth, the SCN of four MR-DHT animals essentially remained devoid of 5-HT fibers. The SCN of two cases with incomplete lesions contained 5-HT-IR fibers. In one of these, the number of
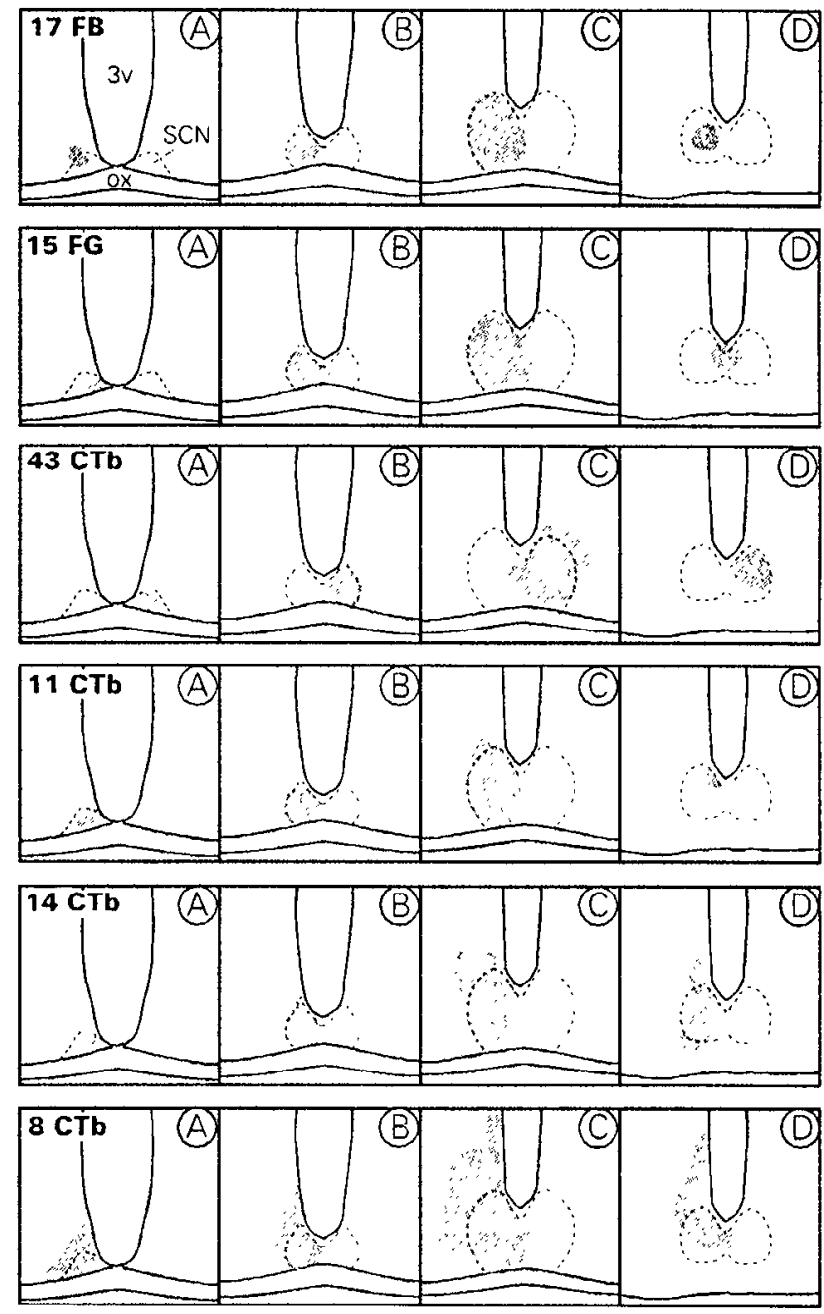

Figure 2. Camera lucida drawings of retrogradc tracer spread throughout the rostrocaudal extent $(A-D)$ of individual SCN injections. The case number and tracer used are indicated at the top of each panel. 


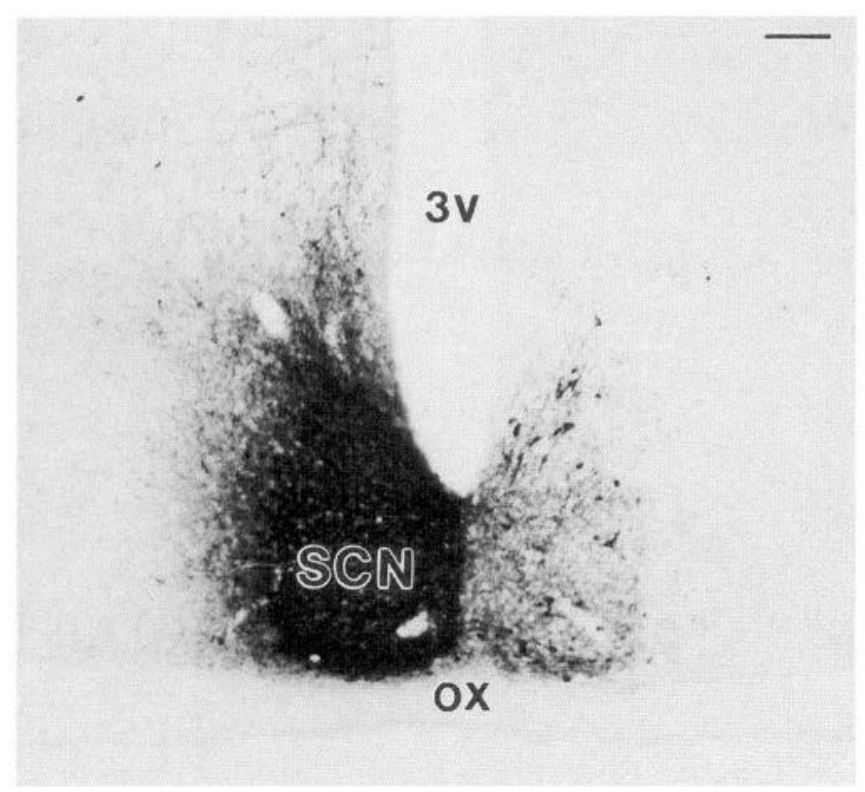

Figure 3. Photomicrograph of the SCN injection site in case 11. There is no spread, only neuronal transport, of CTb to the contralateral SCN or adjacent hypothalamus. Scale bar, $100 \mu \mathrm{m}$.

fibers was reduced but abnormally thick in appearance, an indicator of probable neurotoxic trauma (O'Hearn et al., 1988; Smale et al., 1990). The SCN of the other MR-DHT animal looked normal. Two MR-DHT animals had DHT injections that completely missed the MR. These individuals had SCN 5-HT-IR fiber distribution and density similar to those of CON animals. The SCN of DR-DHT animals had 5-HT-IR fiber distributions identical to those of CON animals. The average 5-HT-IR ROD in the SCN was significantly less in MR-DHT than in CON $(p<0.005)$ or DR-DHT animals $(p<0.005$ ) (Fig. 8), but only if the two MR-DHT animals with undamaged MR are excluded from the comparisons. The SCN ROD in the DR-DHT group did not differ significantly from that in the CON group. The density of 5-HT-IR in the SCN across the brains of all three groups was correlated with the number of 5-HT-IR cells in the MR $(r=0.84 ; p<0.001$; $n=17)$. The number of 5 -HT-IR cells in the DR did not correlate with the 5-HT-IR fiber density in the SCN $(r=0.37 ; p<0.15 ; n$ $=17$ ).

Of the four DR-DHT animals whose brains were analyzed, only one still had a complete loss of fibers in the IGL at the end of the experiment. Two animals had fibers in the IGL, but they looked abnormally thick. The remaining animal had apparently normal 5-HT-IR fibers in the IGL. This animal also had the most DR 5-HT-IR cells remaining in this group. The two MR-DHT animals whose lesions missed the MR had lesions primarily in the left DR, and no fibers remained in the left IGL. Two additional cases had MR lesions that not only destroyed the MR, but also caused bilateral damage to the fibers exiting the DR. There were no remaining fibers in the IGL in either case. In the four remaining MR-DHT animals, fibers exiting laterally from the left DR were damaged, and fibers in the left IGL were also reduced. Spread of the FG-IR along the injection needle track suggests that this damage was probably the result of neurotoxin leakage during syringe withdrawal.

\section{Activity onset, offset, and duration}

DHT injections administered directly into the MR rapidly and substantially altered the hamster running-wheel pattern. In con-
14 FG

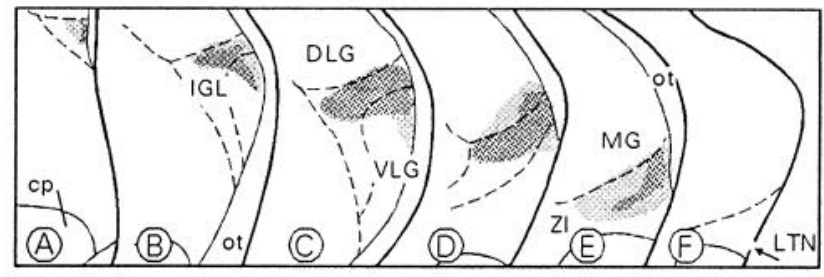

46 CTb

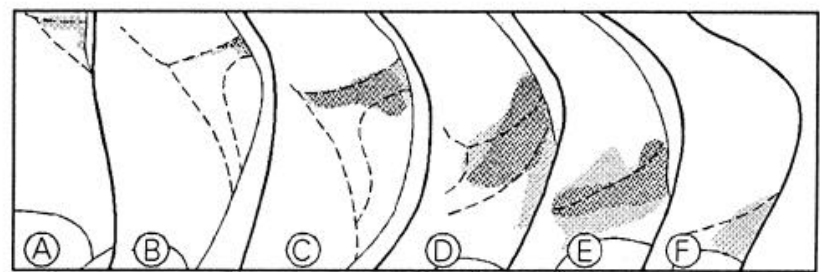

31 CTb

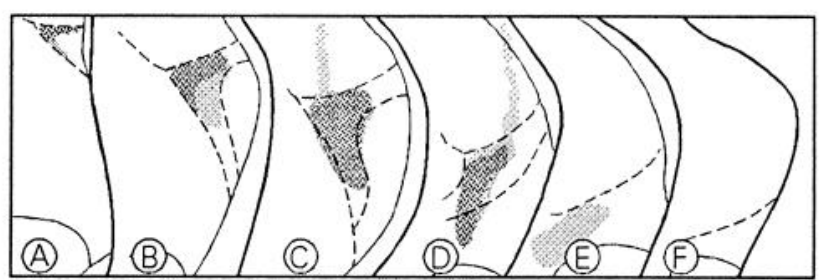

39 CTb

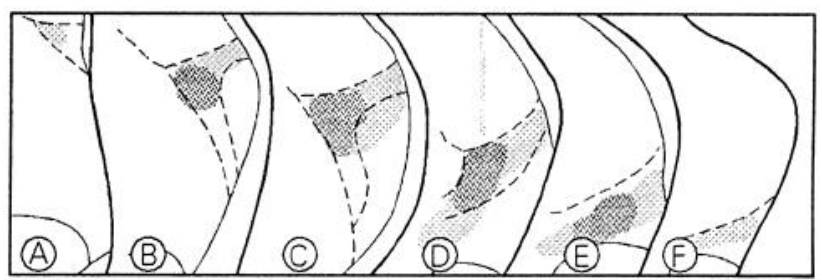

Figure 4. Camera lucida drawings of the rostrocaudal extent of retrograde tracer spread in individual IGL $(A-F)$ injections. Dark shading denotes dense core of injection site. The case number and tracer used are indicated at the top of each panel.

trast, DHT was not effective in changing circadian parameters when applied to the DR (Fig. 9). Figure 10 summarizes the postlesion changes in activity onset, offset, and duration for all groups. Despite the robustness of these behavioral changes, the analysis is conservative because the two MR-DHT animals with missed lesions had behavior similar to that of CON animals, yet they are included for statistical purposes with the MR-DHT group (see Materials and Methods). The DR-DHT group did not differ from the CON group on any measure at any time point analyzed. Changes in activity onset and offset were significantly different between MR-DHT and CON groups $\left(F_{(1,25)}=23.21, p<0.001\right.$; $\left.F_{(1,25)}=5.16, p<0.04\right)$ and between MR-DHT and DR-DHT groups $\left(F_{(1,25)}=12.99, p=0.001 ; F_{(1,25)}=7.51, p=0.01\right)$. As a result of earlier onsets and later offsets, MR-DHT animals had significantly longer $\alpha$ compared with that of $\operatorname{CON}\left(F_{(1,25)}=8.35\right.$, $p<0.01)$ and DR-DHT groups $\left(F_{(1.25)}=9.25, p<0.005\right)$. These lesion effects persisted after surgery, although increased variability in activity offset rendered this measure not significant at postsurgical week 6 . As a consequence, the $\alpha$ of MR-DHT animals was only marginally different at this time compared with $\operatorname{CON}\left(F_{(1,25)}=4.16, p=0.05\right)$ and DHT-DR groups $\left(F_{(1,25)}=\right.$ $4.27, p=0.05)$. Activity onset remained robustly earlier in MR- 
Table 3. Density of PHAL-IR fibers as a result of tracer injection into the MR

Cases receiving PHAL in the MR

\begin{tabular}{|c|c|c|c|c|c|c|c|c|}
\hline & 14 & 1 & $22^{a}$ & 15 & $4^{b}$ & $9^{h}$ & $11^{b}$ & 8 \\
\hline SCN & ++++ & +++ & +++ & ++ & \pm & \pm & \pm & - \\
\hline IGL & \pm & \pm & - & \pm & - & - & \pm & \pm \\
\hline
\end{tabular}

Cases receiving $\mathrm{PHAL}$ in the $\mathrm{DR}$

\begin{tabular}{|c|c|c|c|c|c|c|c|}
\hline & 7 & 24 & $6^{b}$ & $12^{b}$ & $29^{c}$ & $23^{\prime \prime}$ & $25^{d}$ \\
\hline SCN & \pm & \pm & - & - & \pm & - & ++ \\
\hline IGL & ++++ & ++++ & +++ & ++ & \pm & + & +++ \\
\hline
\end{tabular}

-, No fibers present; \pm , fewer than 10 fibers; ++++ , maximal observed PHAL fiber density.

a PHAL sprcad into ventrocaudal DR and DTg.

'PHAL spread did not incorporate entirc nucleus.

'Considerably fewer PHAL fibers throughout the brain compared with similar injections.

${ }^{d}$ PHAL spread into dorsal MR.

DHFT animals compared with CON $\left(F_{(1,25)}=22.87, p<0.001\right)$ and DR-DHT animals $\left(F_{(1.25)}=16.44, p<0.001\right)$.

The variability in activity offset increased further after animals were exposed to $\mathrm{LL}$ conditions. The mean changes in $\alpha$ from presurgical values remained nearly constant for the MR-DHT animals (Fig. 10). The increased variability in LL, however, particularly among CON and DR-DHT animals, eliminated a significant effect of treatment $\left(F_{(2,22)}=1.98, p=0.16\right.$, ANOVA). The running-wheel activity of one MR-DHT animal became disrupted early after transfer to LL, and an accurate measurement of $\alpha$ could not be obtained. This animal was necessarily excluded from $\alpha$ and period analysis in LL.

\section{Circadian periodicity}

Normal circadian rhythmicity of MR-DHT animals was disrupted rapidly during exposure to $\mathrm{LL}$. This disruption ranged from classic "splitting" to approximate arrhythmicity (Fig. 9). By day 25 in LL, one-third of MR-DHT animals (3 of 9) and no animals from DR-DHT or CON groups exhibited disruption in activity. On day 100 of LL, $89 \%(8 / 9)$ of MR-DHT animals, $86 \%(6 / 7)$ of DRDIIT animals, and $63 \%(5 / 8)$ of CON animals displayed a disruption of circadian rhythmicity. A disruption of rhythmicity more extreme than splitting was observed in a significantly greater number of MR-DHT (5/9) than of CON $(0 / 8 ; p=0.02$, Fisher's exact probability test $)$ or DR-DHT animals $(0 / 7 ; p=0.03$, Fisher's exact probability test). Survival analysis showed that the rate of disruption of normal circadian periodicity was significantly quicker in MR-DHT animals $(n=9)$ as compared with CON animals ( $n=10 ; p=0.03$, Gehan's Wilcoxon Test). The DRDHT group $(n=7)$ was not different from CON or MR-DHT groups on this measure $(p<0.15)$. A correlation analysis of all animals whose activity became disrupted in LL showed that an earlier activity onset in LD is associated with an earlier onset of this disruption $(r=0.49 ; p<0.04 ; n=19$ ).

Difterences in circadian period were not evident after $10 \mathrm{~d}$ in LL (CON, 24.27 \pm 0.03 ; MR-DHT, $24.26 \pm 0.03$; DR-DHT, 24.24 $\pm 0.04 ; F_{(2.23)}=0.26, p<0.80$, ANOVA).

\section{Behavioral experiment 2}

\section{Histology: lesion sites and 5-HT cell body destruction}

Thirteen animals had successful MR lesions and are included in all anatomical and behavioral group analyses. In six of these cases $(4,17,23,42,62$, and 66$)$, neither the DR nor the CLi contained
FG-IR and only the ventral aspect of the caudal DR of case 64 had FG-IR. In cases 4 and 17, FG was found in the ventral CLi. Three cases $(3,43$, and 57$)$ with successful MR lesions also had unilateral FG-IR in the DR. Cases 52, 56, and 58 had FG in more than half of the DR. Two additional MR-DHT cases (46 and 47) had FG-spread in less than half of the MR and were eliminated from subsequent anatomical and behavioral group comparisons. All ten lesions aimed at the DR were placed successfully, with no FG-IR found in the MR. FG-IR indicated that in three cases $(20,59$, and 61$)$ the lesions were centered in the caudal DR. Four other cases $(9,45,48$, and 51) had lesion placement in the central region of the DR. Cases 18 and 21 had placements slightly lateral to the midline in which FG did not spread into the most lateral DR "wing" contralateral to the injection site. In case 18 , the lesion was placed in the extreme caudal DR and did not include the rostral end of the nucleus. The precise rostrocaudal placement of the lesion was variable. The majority of the animals in both groups, however, had lesions limited to midbrain and pontine structures.

Neurotoxic lesions of the MR significantly reduced the number of 5-HT-IR neurons in the MR group compared with those in the CON group (Fig. 11) $(t=13.65 ; p<0.001)$. DHT application to the MR caused an $87 \%$ reduction in 5-HT-IR cell number. Similarly, application of DHT to the DR significantly reduced the number of 5-HT-IR cells in the DR compared with those in the CON group (Fig. 11) $(t=6.17 ; p<0.001)$. DHT resulted in a $68 \%$ reduction of 5-HT-IR cells in the DR. Three cases (MR-DHT cases 42 and 43 and DR-DHT case 45) were not included in the cell count analysis because brain sections processed for 5-HT-IR using the monoclonal antibody were unavailable.

\section{5-HT-IR fiber distribution in the SCN and IGL}

Ihe ROD of 5-HI tibers in the SCN was significantly reduced in the MR-DHT group (Fig. 12) compared with the DR-DHT $\left(F_{(1,27)}=99.6, p<0.001\right)$ and CON groups $\left(F_{(1,27)}=9.3, p<\right.$ $0.005)$. Animals with successful DHT lesions of the MR (13 cases) had a complete loss of 5-HT-IR fibers in the SCN (Fig. 13C). The SCN of cases 46 and 47 , which had only partial lesions, still contained 5-HT-IR fibers, although they were less numerous than in CON animals and were thick in appearance. The SCN of DR-DHT animals contained a dense 5-HT-IR fiber plexus distributed similarly to that observed in SCN of CON animals (Fig. $13 B$ ). Interestingly, the SCN-ROD was significantly greater in 

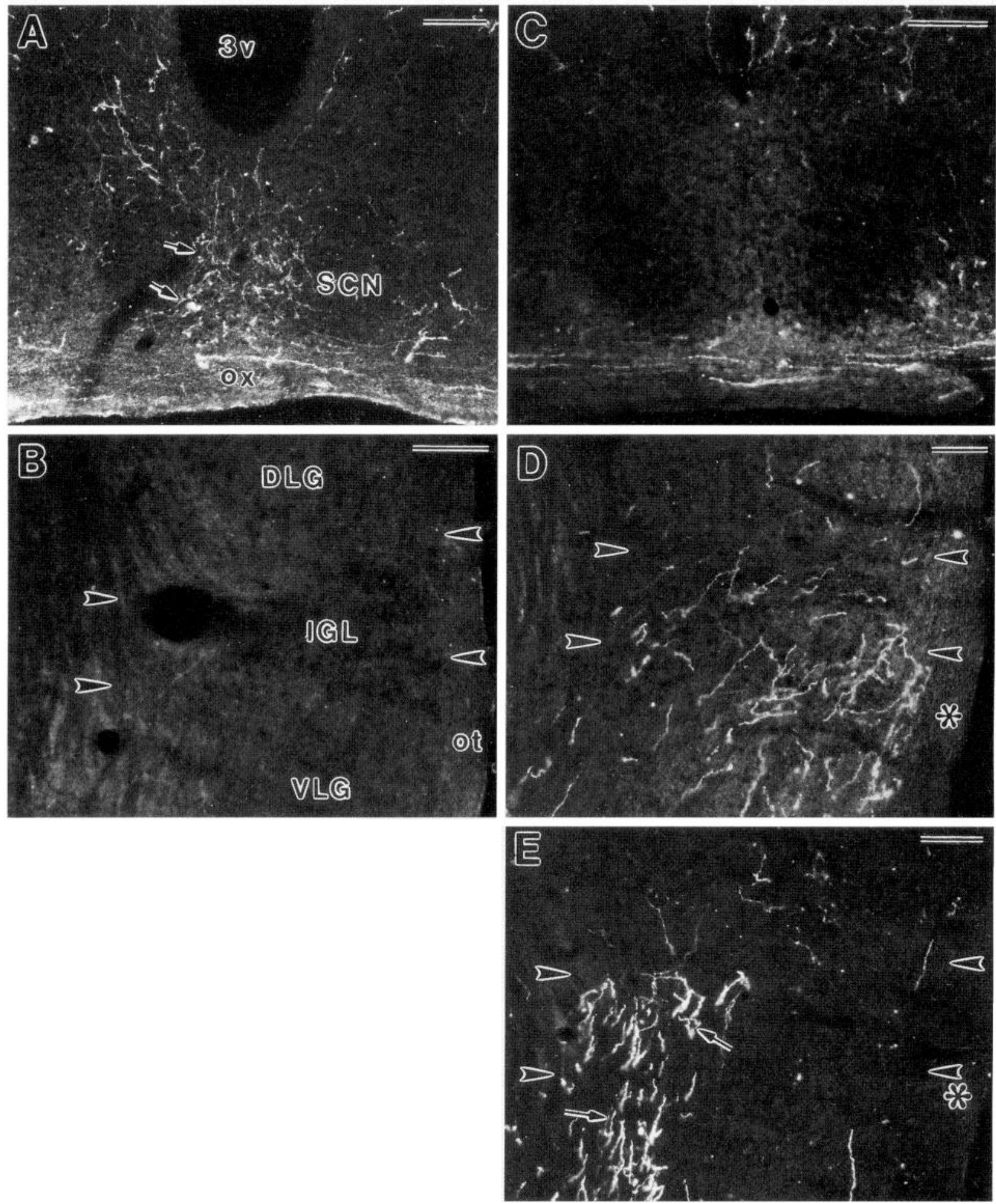

Figure 5. Dark-field photomicrograph of PHAL-IR fiber distribution in the SCN and the IGL. In case 14, a PHAL injection in the MR resulted in fibers located in the SCN $(A)$, primarily in the ventral and medial regions of the nucleus (arrows). Very few fibers were found in the IGL of case $14(B)$. Application of PHAL to the DR of case 7 did not label fibers in the SCN $(C)$; however, PHAL-IR fibers were found to be homogeneously distributed throughout the VLG and IGL in case $7(D)$. In DR case $24(E)$, PHAL-IR fibers were found in both the vIGL and the medial half of the IGL (arrows), but were absent from the lateral half of the IGL (for details, see Results, Anterograde analysis). Arrowheads denote IGL boundaries and asterisks indicate optic tract. Scale bars, $100 \mu \mathrm{m}$.

DR-DHT animals compared with CON animals (Fig. 12) $\left(F_{(1,27)}\right.$ $=31.5, p<0.001)$. Correlation analysis showed a positive relationship between the number of 5-HT-IR cells in the MR and the ROD of 5-HT-IR fibers in the SCN $(r=0.70 ; p<0.001 ; n=27)$.
MR cell counts did not differ between CON and DR-DHT groups. In contrast, the number of 5-HT-IR cells in the DR did not significantly correlate with the ROD of 5-HT-IR fibers in the SCN $(r=0.09 ; p=0.65 ; n=27)$. 

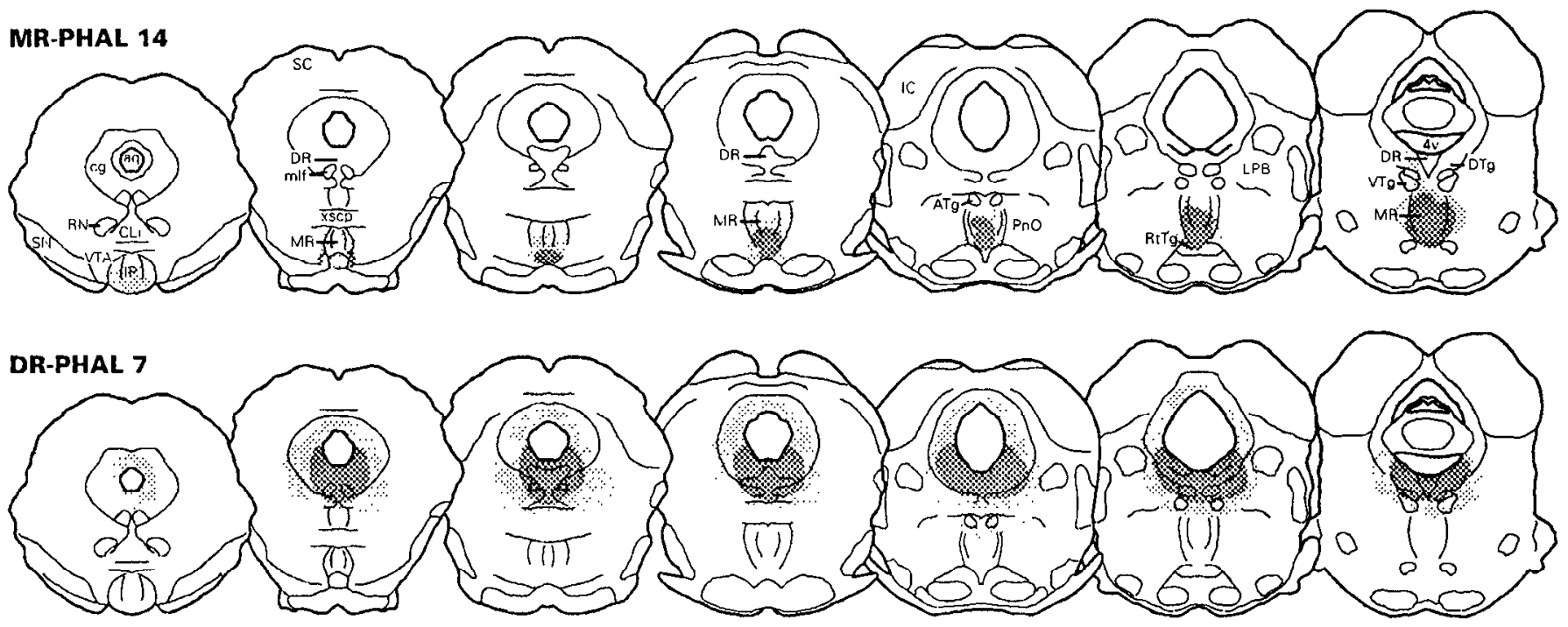

Figure 6. Camera lucida drawings of PHAL tracer injection sites in the MR (top) or the DR (bottom). Dark shading represents core of injection site in which cells were found. Light shading represents areas where tracer spread, but reaction product was light and contained no labeled cells.

Application of DHT to the DR greatly reduced 5-HT-IR fibers in the IGL compared with those in CON brains (Fig. 13E), and no DR-DHT animal had normal 5-HT fiber density and distribution in the IGL. Five animals (cases 9, 45, 48, 51, and 61) had almost a complete elimination of 5-HT-IR fibers in the IGL, with fewer than 10 fibers remaining. Four animals (cases 18, 20, 21, and 59) had slightly more 5-HT fibers remaining, and case 67 had the most 5-HT fibers spared in the DR-DHT group.

MR-DHT animals could be divided into four categories on the basis of 5-HT-IR fiber distribution in the IGL. Four animals (cases 42,62, 64, and 66) had normal densities and distribution of 5-HT-IR fibers in the IGL, with no apparent damage of 5-HT-IR fibers exiting the DR (Fig. 13). MR-DHT cases 3, 43, and 57 had unilateral depletion of 5-HT fibers in the IGL and normal fibers in the contralateral IGL. The 5-HT-IR fibers exiting the DR laterally were unilaterally destroyed, and some DR cell loss was apparent and limited to the side ipsilateral to fiber destruction. Three additional MR-DHT cases (4, 17, and 23) had no evidence of FG spread into the DR, yet had a reduction of 5-HT-IR cells in

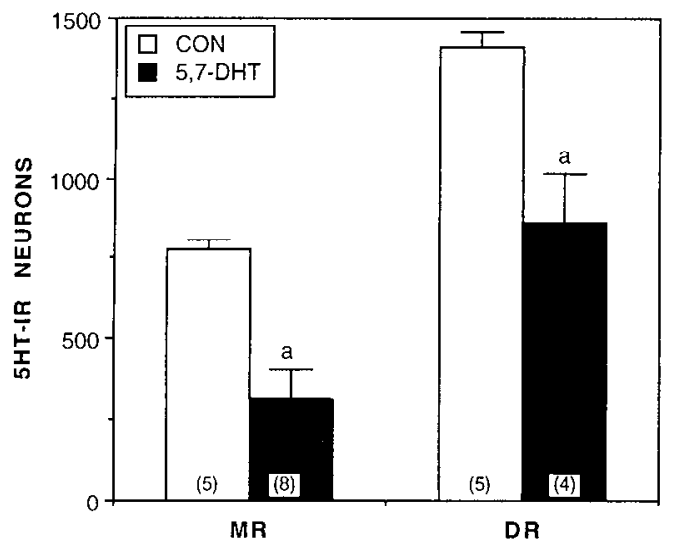

Figure 7. Effect of an injection of vehicle (CON) or 5,7-DHT into the $M R$ or $D R$ on 5 -HT cell counts (mean $\pm \mathrm{SE}$ ) in the respective lesion location. Data from animals in experiment 1 surviving $\sim 36$ weeks postsurgery. The number of animals in each group is in parentheses. a, Significantly different from CON group. the DR and fibers in the IGL. Further visual analysis of these cases revealed that DHT application to the MR was associated with damaged fibers coursing both ventrally and laterally from the DR and with eell loss in the ventral and lateral regions of the DR. Three MR-DHT cases $(52,56$, and 58) with FG-IR in the DR had no remaining 5-HT-IR fibers in the IGL.

\section{Activity onset, offset, and duration}

DHT application to the MR resulted in an advance of activily onset, a delay of activity offset, and a lengthening of $\alpha$ (Fig. 14). The pre- to postsurgical advance in activity onset was significantly greater in MR-DHT animals compared with DR-DHT $(0.81 \pm$ 0.15 vs $\left.0.06 \pm 0.04 \mathrm{hr} ; F_{(1,27)}=20.3, p<0.001\right)$ and CON animals $\left(0.81 \pm 0.15 \mathrm{vs}-0.08 \pm 0.11 \mathrm{hr} ; F_{(1,27)}=22.9, p<0.001\right)$. The delay in activity offset was significantly greater in MR-DHT hamsters compared with the DR-DHT $(1.91 \pm 0.35$ vs $0.23 \pm 0.45 \mathrm{hr}$; $\left.F_{(1.27)}=7.32, p<0.02\right)$ and $\mathrm{CON}$ groups $(1.91 \pm 0.35$ vs $0.32 \pm$

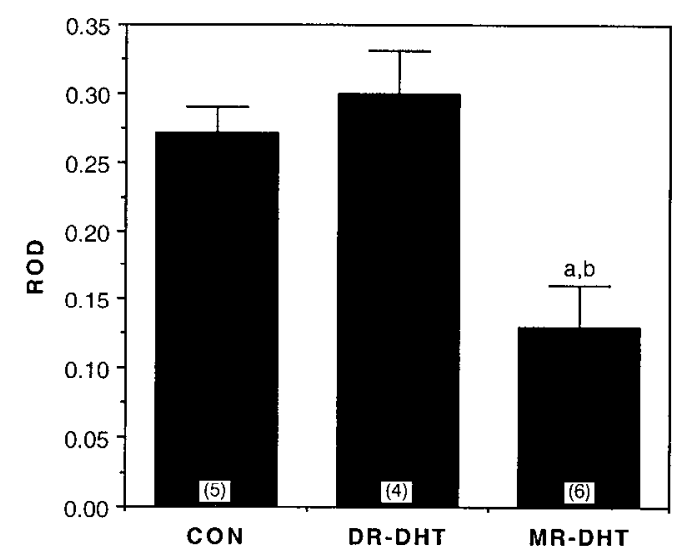

Figure 8. The relative optical density (mean \pm SE) of 5-HT-IR in the SCN in animals from experiment 1 receiving an injection of vehicle $(C O N), 5,7-\mathrm{DHT}$ into the DR (DR-DHT), or 5,7-DHT into the MR $(M R-D H T)$. Survival time was $\sim 36$ weeks postsurgery. The number of brains per group available for histology is in parentheses. Results exclude the two cases with no damage to the MR. $a$, Significantly different from CON group; $b$, significantly different from DR-DHT group. 

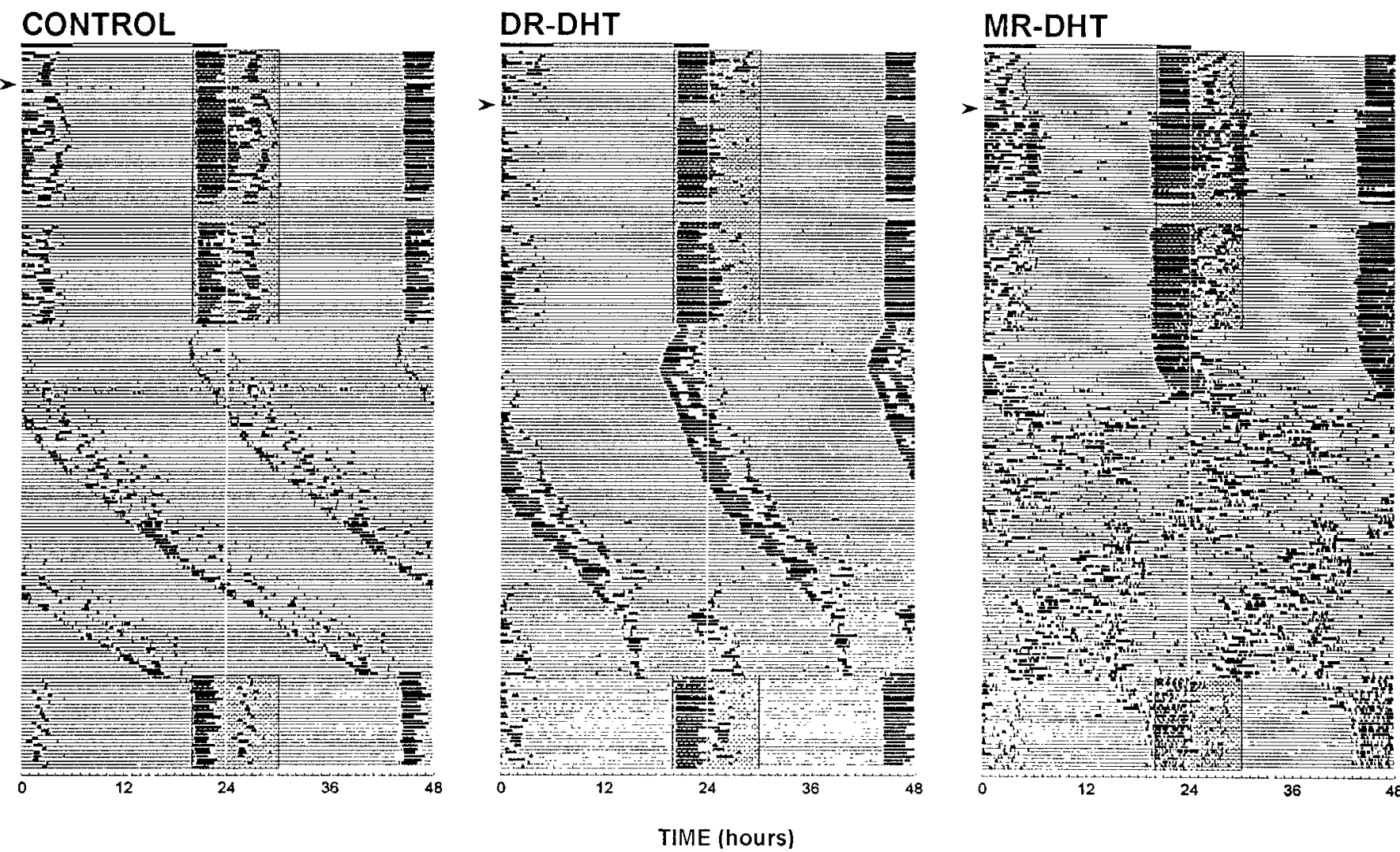

Figure 9. Examples of daily running-wheel activity of animals from experiment 1 (long-term study). Hamsters were initially housed under LDI4:10 (shaded area indicates the time when lights were out) and then transferred to LL for $100 \mathrm{~d}$. Animals were returned to LD14:10 before the termination of the experiment. Left, CONTROL rhythmicity was not affected by an intraraphe vehicle injection (arrowhead) during LD or LL. Middle, A DR-DHT lesion (arrowhead) also had no effect on circadian rhythmicity under LD conditions. This animal exhibited a typical split in its activity phase after 71 d in LL. Right, An MR-DHT lesion (arrowhead) caused an immediate expansion of the activity phase in ID. The behavior of this animal became disrupted on day 21 of LL, after which a clear circadian rhythm was not always evident

$\left.0.71 \mathrm{hr} ; F_{(1,27)}=5.28, p<0.03\right)$. Consequently, $\alpha$ was significantly more expanded in MR-DHT hamsters compared with DR-DHT $\left(2.72 \pm 0.45\right.$ vs $\left.0.29 \pm 0.45 \mathrm{hr} ; F_{(1,27)}=12.9, p<0.001\right)$ and $\mathrm{CON}$ animals $\left(2.72 \pm 0.45\right.$ vs $\left.0.23 \pm 0.69 \mathrm{hr} ; F_{(1,27)}=11.0, p<0.003\right)$. DR-DHT and CON groups were not significantly different on any of these measures (onset, $F_{(1,27)}=0.5, p<0.50$; offset, $F_{(1,27)}=$ $\left.0.02, p<0.90 ; \alpha, F_{(1,27)}=0.01, p<0.95\right)$.

Correlation analysis revealed that the fewer the number of 5-HT cells in the MR $(n=27)$, the greater the advance in activity onset $(r=0.70 ; p<0.001)$, delay in activity offset $(r=0.46 ; p<$ $0.02)$, and increase in $\alpha(r=0.58 ; p<0.002)$. Similarly, the lower the ROD of 5-HT-IR in the SCN $(n=30)$, the greater the advance of activity onset $(r=0.55 ; p<0.003)$, delay in offset ( $n$ $=30 ; r=0.44 ; p<0.02)$, and expansion of $\alpha(n=30 ; r=0.52$; $p<0.003)$.

\section{DISCUSSION}

\section{Neuroanatomical considerations}

Raphe projections to the $S C N$

The present experiments use three anatomical methods to demonstrate that the serotonergic innervation of the SCN originates in the median raphe. (1) Anterograde tracing of MR efferents identifies a fiber pattern in the ventromedial SCN similar to the normal distribution of 5-HT fibers in the SCN (Morin et al., 1992; Card and Moore, 1984); (2) retrogradely labeled cells containing 5-HT are found almost exclusively in the MR after tracer appli- cation to the SCN; and (3) neurotoxic lesions of the MR eliminate 5-HT-IR fibers in the SCN.

The three techniques also provide an internally consistent data set showing that a projection to the SCN, serotonergic or otherwise, does not originate in the DR. The conclusion that the hamster MR, rather than the DR, projects to the $\mathrm{SCN}$ contradicts the prevailing view. This view has been derived largely from rat studies using anterograde autoradiographic techniques that have greater methodological variability and interpretive difficulty than the present methods have (Conrad et al., 1974; Pierce et al., 1976; Azmitia and Segal, 1978; Moore et al., 1978). In general, the pattern of serotonergic basal forebrain innervation is similar in both developing and adult rat and hamster (Steinbusch, 1981; Lidov and Molliver, 1982a,b; Wallace and Lauder, 1983; Botchkina and Morin, 1993), making an SCN species difference unlikely. More importantly, the present hamster data are supported directly by recent PHAL analysis of rat forebrain innervation, which also failed to demonstratc a projection to the SCN by DR neurons (Vertes, 1991). In addition, preliminary PHAL data show a projection from the rat MR to the SCN (Mnga and Moore, 1994). The present data also suggest that previously observed retrogradely labeled DR cells (Pickard, 1982; Bina et al., 1993) are the result of tracer spread outside the SCN.

Previous lesion studies from this laboratory implied a causal link between the 5-HT neuronal loss in the DR and fiber loss in the SCN (Smale et al., 1990; Morin and Blanchard, 1991a,b). It 


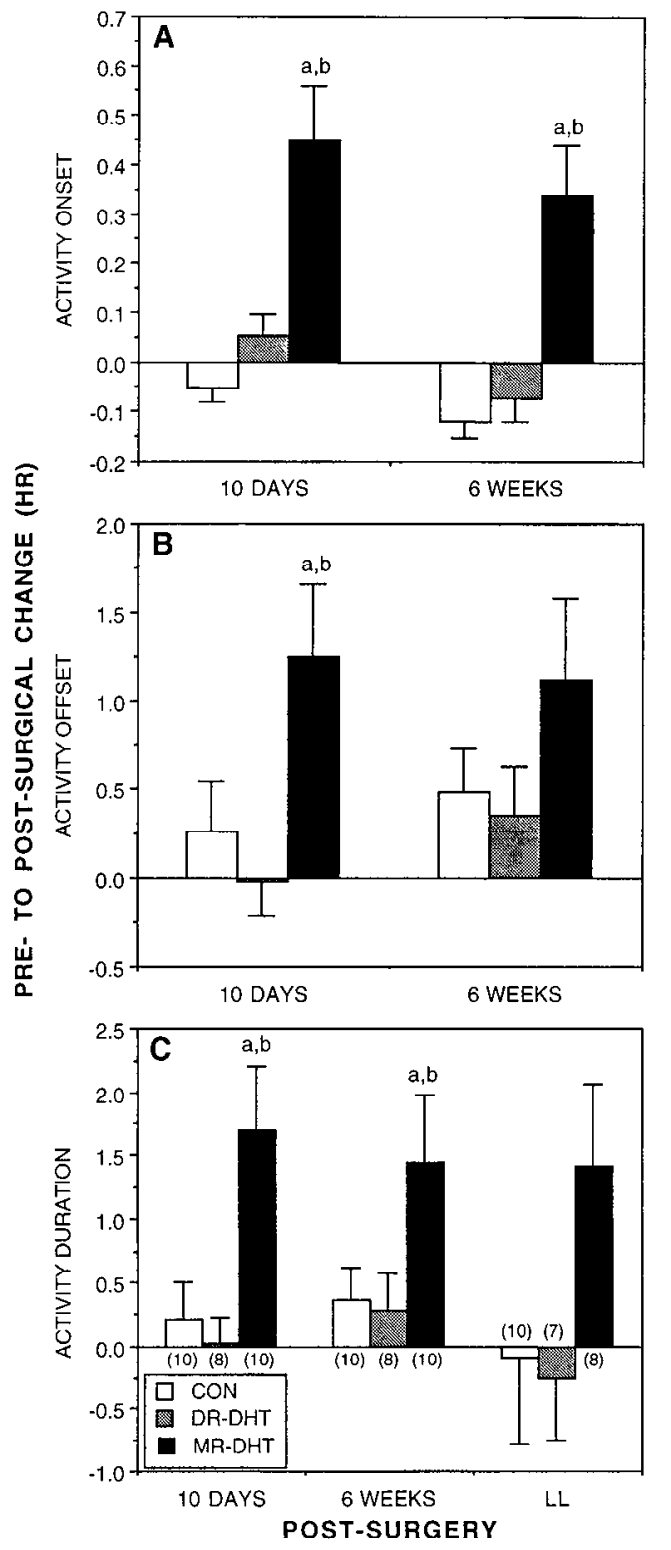

Figure 10. The pre- to postsurgical change (mean $\pm \mathrm{SE}$ ) in activity onset $(A)$, oftset $(B)$, and duration (C) calculated $10 \mathrm{~d}$ and 6 weeks postsurgery in animals housed under LD14:10. Pre- to postsurgical change in activity duration was also measured after at least $10 \mathrm{~d}$ in LL. Comparisons were made between animals with intraraphe vehicle injections (CON), 5,7-DHT lesions of the DR (DR-DHT), or 5,7-DHT lesions of the MR (MR-DHT). The number of animals in each group is shown in parentheses. $a$, Significantly different from CON group; $b$, significantly different from DR-DHT group.

now seems that these changes occurred because DR and SCN were damaged independently by intraventricular application of neurotoxin. The regeneration of 5-HT fibers in the SCN after intraventricular neurotoxin (Bosler et al., 1992; Morin, 1992) suggests that this method of delivery may not destroy many of the MR serotonergic cell bodies projecting to the SCN. Such an interpretation is supported by the present data showing that direct DHT application to the MR did not result in fiber regeneration in the SCN. The data also indicate the presence of relatively small numbers of SCN afferent 5-HT neurons in the MR, the precise locations of which are difficult to identify across animals. Therefore, it is unlikely that previous studies using intraventricular

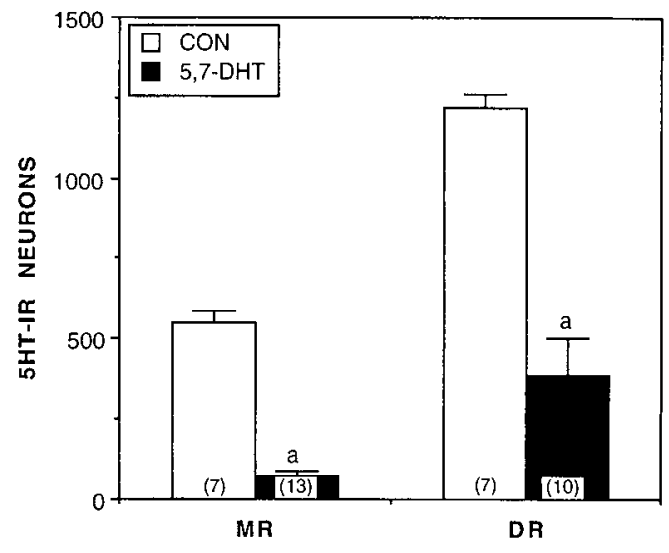

Figure 11. In experiment 2 , the acute effect (7-10 d postsurgery) of 5,7-DHT application to the DR or MR on 5-HT-IR cell counts (mean \pm $\mathrm{SE})$ is compared with vehicle-injected animals $(C O N)$. The number of animals in each group is in parentheses. $a$, Significantly different from CON group.

DHT could have identified which neurons innervate the SCN solely on the basis of a small number missing from the MR. Furthermore, the fact of regeneration after intraventricular DHT suggests that the critical MR neurons were disconnected from the SCN but not eliminated from the brain. The apparently small numbers of widely distributed SCN afferent neurons may also account for the large variability in PHAL-IR fibers in the SCN.

\section{Raphe projections to the IGL}

A serotonergic projection to the IGL originating in the DR was demonstrated directly using complementary retrograde and anterograde techniques and is generally consistent with previous studies of the lateral geniculate complex (Pierce et al., 1976; Azmitia and Segal, 1978; Pasquier and Villar, 1982; Villar et al., 1988; Vertes, 1991; Waterhouse et al., 1993). Moreover, the present data are in accord with reports showing that the projection to the LGN originates primarily in the rostral half of the DR, with little contribution from the caudal DR (Waterhouse et al., 1993). The present study did not find regional specificity of labeled cells within the anterior DR, as described previously (Pas-

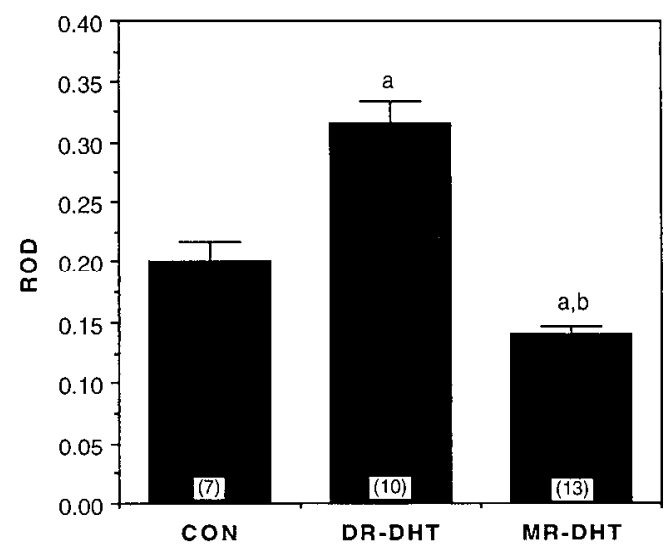

Figure 12. The relative optical density (mean \pm SE) of 5-HT-IR in the SCN of animals in experiment 2 with intraraphe vehicle injections $(C O N)$, $5,7-\mathrm{DHT}$ injections into the DR (DR-DHT), or 5,7-DHT injections into the MR $(M R-D H T)$. The number of animals in each group is in parentheses. $a$, Significantly different from CON group; $b$, significantly different from DR-DHT group. 

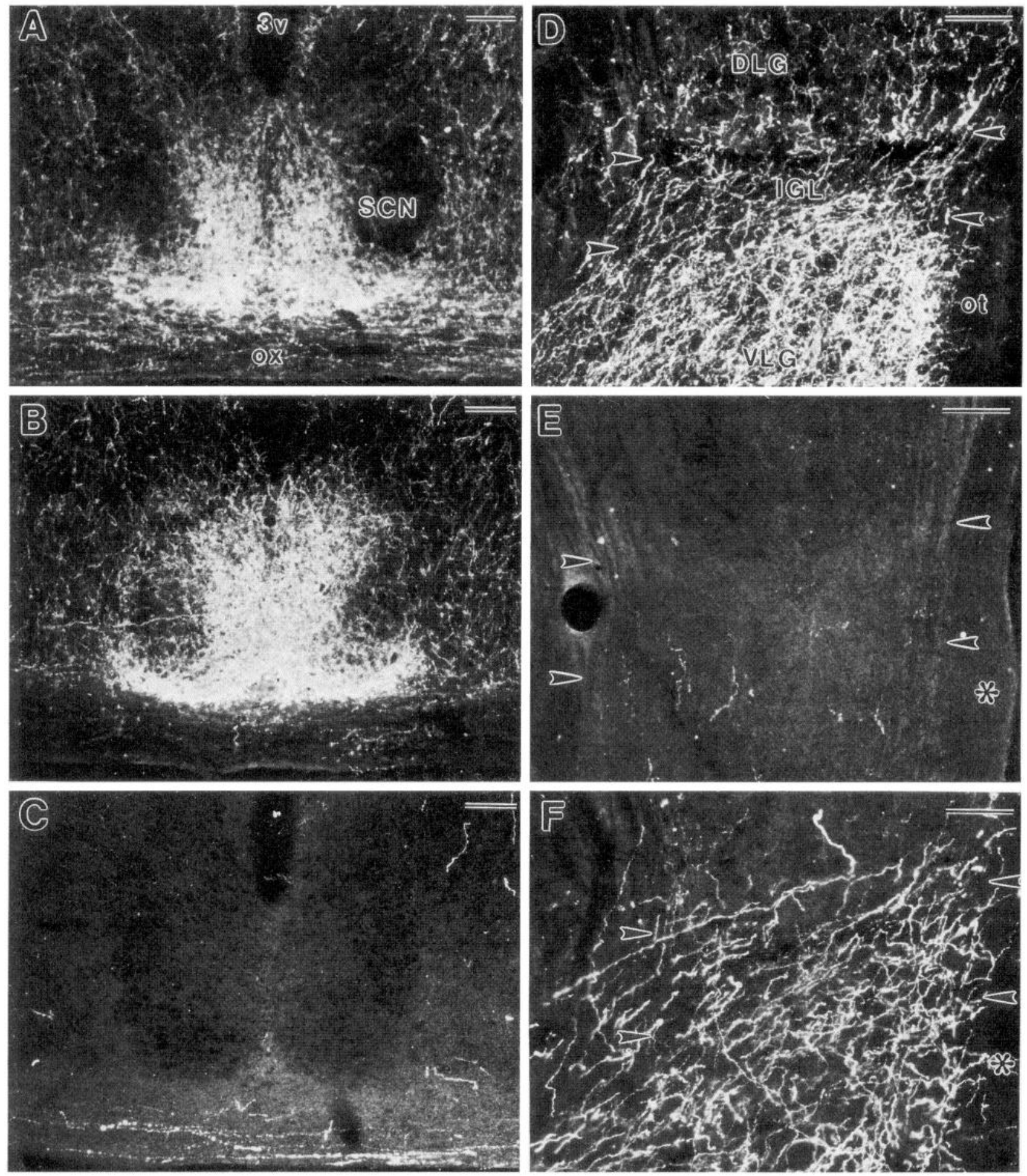

Figure 13. Dark-field photomicrographs of 5-HT-IR fibers remaining in the SCN $(A)$ and IGL $(D)$ of a CON animal treated with an intraraphe vehicle injection; in the SCN $(B)$ and IGL $(E)$ of case 51 with a DHT injection into the DR; and in the SCN $(C)$ and IGL $(F)$ of MR-DHT case 66. Arrowheads denote IGL boundaries and asterisks indicate optic tract. Scale bars, $100 \mu \mathrm{m}$.

quier and Villar, 1982; Villar et al., 1988; Waterhouse et al., 1993). This may be related to the small size of the injections, which labeled fewer cells compared with those in previous reports (Villar et al., 1988; Waterhouse et al., 1993).

The present anterograde data provide evidence for innervation of the IGL by the DR via two distinct pathways, both of which are necessary for normal 5-HT-IR fiber distribution in the LGN complex. One innervates the ventral IGL and the medial half of the IGL at the level of its "leaflet" configuration and is consistent with the arcuate tract described in rats (Azmitia and Segal, 1978). A second pathway originates primarily in the rostral DR and innervates the lateral VLG and IGL. This pathway has an origin similar to that for the periventricular tract in the rat (Azmitia and Segal, 1978). Although a projection to the LGN via this pathway has not been identified in the rat (Azmitia and Segal, 1978), this issue has not been investigated fully. 

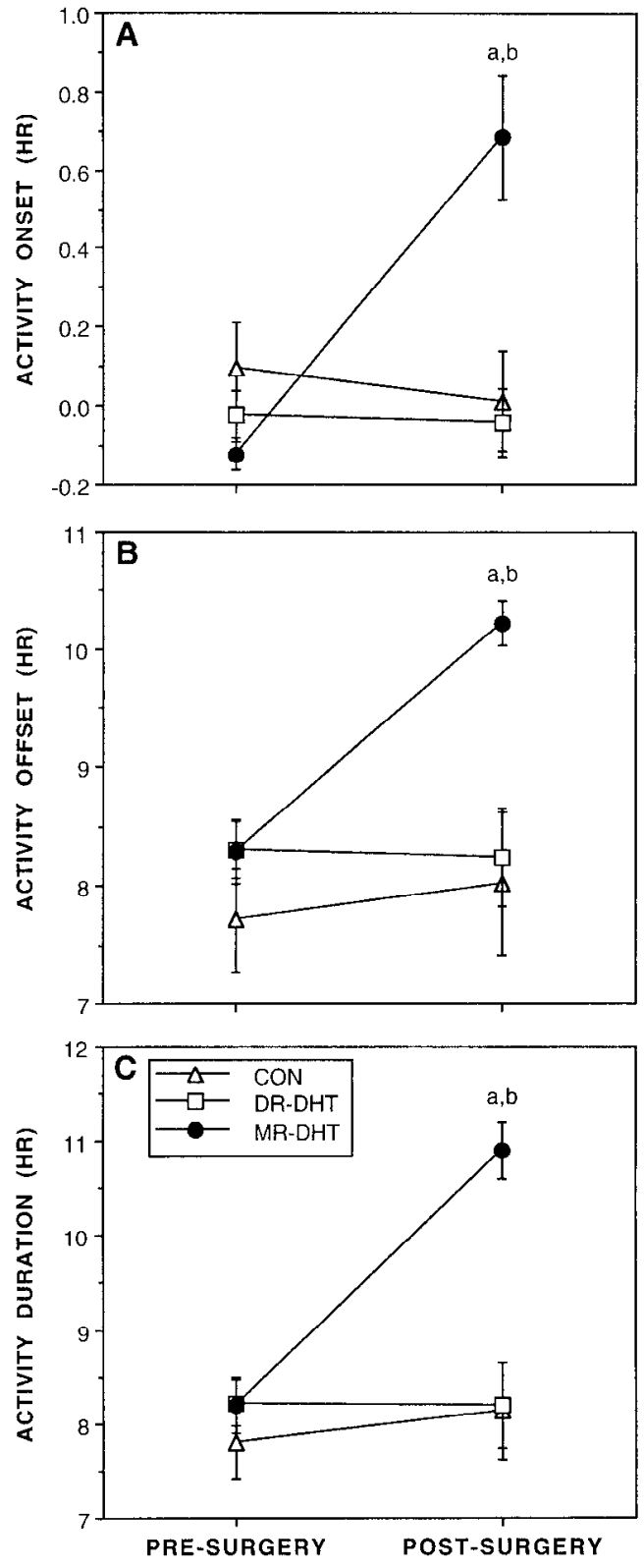

Figure 14. The acute affect (7-10 d postsurgery) of 5,7-DHT application to the MR or the DR on activity phase onset $(A)$, offset $(B)$, and duration (C) in animals housed under LD14:10 (experiment 2). $a$, Significantly different from CON group; $b$, significantly different from DR-DHT group.

The present lesion data provide additional support for the view that the 5-HT innervation of the LGN complex originates in the DR (Pasquier and Villar, 1982; Villar et al., 1988). DHT application directly to the DR eliminated 5-HT-IR fibers in the IGL. MR destruction resulted in fiber depletion in the IGL only when the fibers making a lateral exit from the DR were also destroyed. The lesion results are consistent with the failure to demonstrate $M R$ innervation of the IGL (present data) (Azmitia and Segal, 1978; Vertes and Martin, 1988; Vaudano and I egg, 1992).

Individual raphe neurons are known to have multiple targets (DeOlmos and Heimer, 1980; Imai et al., 1986; Villar et al., 1988; Li et al., 1993a,b; Losier and Semba, 1993). The reduction of 5-HT-IR fibers in the IGL as a consequence of DHT application to the DR never occurred independent of a similar reduction in the VLG. Therefore, it is possible that one set of cells innervates both the IGL and other proximal structures in the visual system. This possibility cannot be excluded by the retrograde data analysis because of the difficulty in containing the tracer solely within the boundaries of the IGL. All three anatomical techniques used in the present study, however, support the view that the SCN and IGL are innervated independently by midbrain raphe neurons and as a consequence are specifically not innervated by the same serotonergic neurons.

The present data show both 5-HT and non-5-HT pathways to the SCN and IGL from the MR and the DR, respectively. Serotonergic and nonserotonergic DR projections to the rat LGN have also been described (Villar et al., 1988), although similar studies of the SCN have not been performed previously. Neurons in the raphe contain various neuromodulators (Nanopoulos et al., 1982; Sutin and Jacobowitz, 1988; $\mathrm{Li}$ et al., 1990), but their relative contribution to the projections to the SCN or IGL has not been investigated. We cannot eliminate the possibility that medullary raphe nuclei also innervate the SCN and the IGL. Although these nuclei, particularly the raphe magnus, may project rostrally (Bobillier et al., 1976; Peschanski and Besson, 1984; Vertes, 1984; Sim and Joseph, 1992), it is not clear that these projections are serotonergic (Kohler et al., 1982; Bowker, 1986). Moreover, the use of lesions that specifically target 5-HT neurons in the MR and DR affirms the conclusions suggested by the present anatomical analysis.

\section{Functional considerations}

Intraventricular administration of DHT induces changes in circadian regulation of the locomotor rhythm (Smale et al., 1990; Morin and Blanchard, 1991a,b; Morin, 1992). The present data support the view that the scrotoncrgic ncurons whose efferents regulate circadian function are in the MR and specifically exclude a role for the DR. The changes in entrained activity after MR lesions are similar to those seen after widespread 5-HT depletion (Smale et al., 1990; Morin and Blanchard, 1991b). In addition, only lesions of the MR could account for both the quantitative and qualitative disruption of circadian rhythmicity reported previously in 5-HT-depleted animals housed in LL (Morin and Blanchard, 1991b). The MR-DHT animals with the largest changes in LD had both the most disrupted rhythmicity in LL and the earliest onset of disruption, suggesting that the MR modulates circadian behavior in both LD and LL. The extreme types of noncircadian behavior seen after MR (present data) or general 5-HT depletion (Morin and Blanchard, 1991b) were never seen in either CON or DR-DHT animals.

The foregoing indicates the substantial similarity between the consequences of general brain depletion of 5-HT and the MRtargeted depletion; however, two differences remain and require further explanation. In hamsters given intraventricular DHT, the activity phase remains expanded, and the circadian period is greatly lengthened in LL. In the present study, the activity phase of MR-DHT animals remained expanded and very similar to that seen during LD14:10 but was not significantly different from that in CON animals. The large variability in activity offset during LL in the present study may account for this lack of difference. The failure to find an increase in circadian period by MR-DHT animals may be rclated simply to the necessity of measuring period after only $10 \mathrm{~d}$ in LL.

It is important to emphasize that DHT application to the MR results in the depletion of 5-HT in all MR target nuclei. Because these lesions directly affect the circadian timing system, we conclude that behavioral changes are likely to be the result of $5-\mathrm{HT}$ 
depletion in the SCN. A direct test of this hypothesis has not been conducted, and the possible contribution of other MR-recipient nuclei to the several circadian rhythm changes cannot yet be excluded.

There is no established function of the serotonergic DR projection to the IGL. The IGL has been implicated as a possible pathway through which activity or a correlate thereof may influence the circadian clock (Wickland and Turek, 1994). Phase shifts to nonphotic stimuli, the effects of which have been proposed to be mediated by activity feedback onto the clock (Turek, 1989; Van Reeth and Turek, 1989; Wickland and Turek, 1991; Van Reeth et al., 1993), are eliminated by lesions of the IGL (Johnson et al., 1989; Janik and Mrosovsky, 1994; Wickland and Turek, 1994). Recently, we found that global 5-HT depletion dramatically reduces the induction of acute activity associated with both novel wheel access and triazolam but does not block phase shifts to these stimuli (Meyer and Morin, 1995). Because the DR is rich in benzodiazepine receptors (Michels et al., 1990), the projection from the DR to the IGL may indeed have a role in mediating these phase shifts, but 5-HT does not seem to be necessary for their induction.

In summary, the present data provide exterssive anatomical evidence establishing that the MR, but not the $D R$, projects to the $\mathrm{SCN}$. A serotonergic projection from the MR mediates circadian rhythmicity in both LD and LL conditions. The present experiments also demonstrate a serotonergic projection from the DR to the IGL, the function of which has yet to be elucidated.

\section{REFERENCES}

Azmitia EC (1978) The serotonin-producing neurons of the midbrain median and dorsal raphe nuclei. In: Handbook of psychopharmacology, (Iversen LL, Iversen SD, Snyder SH, eds), pp 233-314. New York: Plenum.

Azmitia EC, Scgal M (1978) An autoradiographic analysis of the differential ascending projections of the dorsal and median raphe nuclei in the rat. J Comp Neurol 179:641-668.

Bina KG, Rusak B, Scmba K (1993) Localization of cholinergic neurons in the forebrain and brainstem that project to the suprachiasmatic nucleus of the hypothalamus in rat. J Comp Neurol 335:295-307.

Bobillier P, Seguin S, Petitjean F, Salvert D, Touret M, Jouvet M (1976) The raphe nuclei of the cat brainstem: a topographical atlas of their efferent projections as revealed by autoradiography. Brain Res 113:449-486.

Bosler O, Vuillon-Cacciuttolo G, Saidi H (1992) Long-term serotonin reinnervation of the suprachiasmatic nucleus after 5,7-dihydroxytryptamine axotomy in the adult rat. Neurosci Lett 143:159-163.

Botchkina GI, Morin LP (1993) Development of the hamster serotoninergic system: cell groups and diencephalic projections. J Comp Neurol 338:405-431.

Bowker RM (1986) The relationship between descending serotonin projections and ascending projections in the nucleus raphe magnus: a double labeling study. Neurosci Lett 70:348-353.

Card JP, Moore RY (1984) The suprachiasmatic nucleus of the golden hamster: immunohistochemical analysis of cell and fiber distribution. Neuroscience 13:390-396.

Card JP, Moore RY (1989) Organization of lateral geniculatehypothalamic connections in the rat. J Comp Neurol 284:135-147.

Conrad LCA, Leonard CM, Pfaff DW (1974) Connections of the median and dorsal raphe nuclei in the rat: an autoradiographic and degeneration study. J Comp Neurol 156:179-206.

DeOlmos J, Heimer L (1980) Double and triple labeling of neurons with fluorescent substances; the study of collateral pathways in the ascending raphe system. Neurosci Lett 19:7-12.

Edgar DM, Miller JD, Prosser RA, Dean RR, Dement WC (1993) Serotonin and the mammalian circadian system. II. Phase-shifting rat behavioral rhythms with serotonergic agonists. J Biol Rhythms 8:17-31.

Glass JD, Randolph WW, Ferreira SA, Rea MA, Hauser UE, Blank JL, De Vries MJ (1992) Diurnal variation in 5-hydroxyindole-acetic acid output in the suprachiasmatic region of the Siberian hamster assessed by in vivo microdialysis: evidence for nocturnal activation of serotonin release. Neuroendocrinology 56:582-590.

Glass JD, Hauser UE, Randolph WW, Rea MA, De Vries MJ (1993) In vivo microdialysis of 5-hydroxyindoleacetic acid and glutamic acid in the hamster suprachiasmatic nuclei. Am Zool 33:212-218.

Glass JD, Selim M, Rea MA (1994) Modulation of light-induced C-Fos expression in the suprachiasmatic nuclei by $5-\mathrm{H}^{\prime} \mathrm{I}_{\mathrm{IA}}$ receptor agonists. Brain Res 638:235-242.

Harrington ME, Rusak B (1986) Lesions of the thalamic intergeniculate leaflet alter hamster circadian rhythms. J Biol Rhythms 1:309-325.

Harrington ME, Rusak B (1988) Ablation of the geniculo-hypothalamic tract alters circadian activity rhythms of hamsters housed under constant light. Physiol Behav 42:183-189.

Harrington ME, Rusak B (1989) Photic responses of geniculohypothalamic tract neurons in the Syrian hamster. Vis Neurosci 2:367-375.

Imai H, Steindler DA, Kitai ST (1986) The organization of divergent axonal projections from the midbrain raphe nuclei in the rat. J Comp Neurol 243:363-380.

Jacobs BL, Azmitia EC (1992) Structure and function of the brain serotonin system. Physiol Rev 72:165-229.

Janik D, Mrosovsky N (1994) Intergeniculate leaflet lesions and behaviorally induced shifts of circadian rhythus. Brain Res 651:174-182.

Johnson RF, Moore RY, Morin LP (1988a) Loss of entrainment and anatomical plasticity after lesions of the hamster retinohypothalamic tract. Brain Res 460:297-313.

Johnson RF, Morin LP, Moore RY (1988b) Retinohypothalamic projections in the hamster and rat demonstrated using cholera toxin. Brain Res 462:301-312.

Johnson RF, Moore RY, Morin LP (1989) Lateral geniculate lesions alter activity rhythms in the hamster. Brain Res Bull 22:411-422.

Klein DC, Moore RY, Reppert SM (1991) Suprachiasmatic nucleus: the mind's clock. New York: Oxford UP.

Kohler C, Chan-Palay V, Steinbusch H (1982) The distribution and origin of serotonin-containing fibers in the septal area: a combined immunohistochemical and fluorescent retrograde tracing study in the rat. $\mathrm{J}$ Comp Neurol 209:91-111.

Li Y, Jia H, Rao Z, Shi J (1990) Serotonin-, substance P- or leucineenkephalin-containing neurons in the midbrain periaqueductal gray and nucleus raphe dorsalis send projection fibers to the central amygdaloid nucleus in the rat. Neurosci Lett 120:124-127.

Li Y, Takada M, Matsuzaki S, Shinonaga Y, Mizuno N (1993a) Identification of periaqueductal gray and dorsal raphe nucleus neurons projecting to both trigeminal sensory complex and forebrain structures: a fluorescent retrograde double-labeling study in the rat. Brain Res 623:267-277.

I.i Y, Takada M, Mizuno N (1993b) Collateral projections of single neurons in the periaqueductal gray and dorsal raphe nucleus to both the trigeminal sensory complex and spinal cord in the rat. Neurosci Lett 153:153-156.

Lidov HGW, Molliver ME (1982a) Immunohistochemical study of the development of serotonergic neurons in the rat CNS. Brain Res Bull 9:559-604.

Lidov HGW, Molliver ME (1982b) An immunohistochemical study of serotonin neuron development in the rat: ascending pathways and terminals fields. Brain Res Bull 8:389-430.

Losier BJ, Semba K (1993) Dual projections of single cholinergic and aminergic brainstem neurons to the thalamus and basal forebrain in the rat. Brain Res 604:41-52.

Mantyh PW, Kemp JA (1983) The distribution of putative neurotransmitters in the lateral geniculate nucleus of the rat. Brain Res 288:344-348.

Medanic M, Gillette MU (1992) Serotonin regulates the phase of the rat suprachiasmatic circadian pacemaker in vitro only during the subjective day. J Physiol (Lond) 450:629-642.

Meijer JH, Groos GA (1988) Responsiveness of suprachiasmatic and ventral lateral geniculate neurons to serotonin and imipramine: a microiontophoretic study in normal and imipramine-treated rats. Brain Res Bull 20:89-96.

Meyer EL, Morin LP (1995) The effects of serotonin depletion on triazolam and novel wheel induced phase shifts. Soc Neurosci Abstr 20:179.

Michels KM, Morin LP, Moore RY (1990) GABA /benzodiazepine receptor localization in the circadian timing system. Brain Res 531:16-24. 
Miller JD, Fuller CA (1990) The response of suprachiasmatic neurons of the rat hypothalamus to photic and serotonergic stimulation. Brain Res 515:155-162.

Moga MM, Moore RY (1994) An anterograde tracing study of neuronal inputs to the suprachiasmatic nucleus in the rat. Soc Neurosci Abstr 20:347.

Moore RY (1973) Retinohypothalamic projection in mammals: a comparative study. Brain Res 49:403-409.

Moore RY, Halaris AE, Jones BE (1978) Serotonin neurons of the midbrain raphe: ascending projections. J Comp Neurol 180:417-438.

Morin LP (1992) Serotonergic reinnervation of the hamster suprachiasmatic nucleus and intergeniculate leaflet without functional circadian rhythm recovery. Brain Res 599:98-104.

Morin LP (1993) Age, but not pincal status, modulates circadian periodicity of golden hamsters. J Biol Rhythms 8:189-197.

Morin LP, Blanchard JH (1991a) Serotonergic modulation of the hamster whecl running rhythm: response to lighting conditions and food deprivation. Brain Res 566:186-192.

Morin LP, Blanchard JH (1991b) Depletion of brain serotonin by 5,7DHT modifies hamster circadian rhythm response to light. Brain Res $566: 173-185$

Morin LP, Blanchard JH, Moore RY (1992) Intergeniculate leaflet and suprachiasmatic nucleus organization and connections in the hamster. Vis Neurosci 8:219-230.

Nanopoulos D, Belin MF, Maitre M, Vincendon G, Pujol JF (1982) Immunocytochemical evidence for the existence of (iABAcrgic neurons in the nuclcus raphe dorsalis: possible existence of neurons containing serotonin and GABA. Brain Res 232:375-389.

O'Hearn E, Battaglia G, DeSouza EB, Kuhar MJ, Molliver ME (1988) Methylenedioxyamphetamine (MDA) and methylenedioxymethamphetamine (MDMA) cause selective ablation of scrotonergic axon terminals in forebrain: immunocytochemical evidence for neurotoxicity. J Neurosci 8:2788-2803.

Pasquier DA, Villar MJ (1982) Specific serotonergic projections to the lateral geniculate body from the lateral cell groups of the dorsal raphe nucleus. Brain Res 249:142-146.

Peschanski M, Besson J (1984) Diencephalic connections of the raphe nuclei of the rat brainstem: an anatomical study with reference to the somatosensory system. J Comp Neurol 224:509-534.

Pickard GE (1982) The afferent connections of the suprachiasmatic nucleus of the golden hamster with emphasis on the retinohypothalamic projection. J Comp Neurol 211:65-83.

Pickard GE, Ralph MR, Menaker M (1987) The intergeniculate leaflet partially mediates effects of light on circadian rhythms. J Biol Rhythms 2:35-56.

Pierce ET, Foote WE, Hobson JA (1976) The efferent connection of the nucleus raphe dorsalis. Brain Res 107:137-144.

Poncet L, Denoroy L, Jouvet M (1993) Daily variations in vivo tryptophan hydroxylation and in the contents of serotonin and 5 -hydroxyindoleacetic acid in discrete brain areas of the rat. J Neural Transm 92:137-150.

Prosser RA, Miller JD, Hcller HC (1990) A serotonin agonist phase shifts the circadian clock in the suprachiasmatic nuclei in vitro. Brain Res 534:336-339.

Prosser RA, Heller HC, Miller JD (1992) Serotonergic phase shifts of the mammalian circadian clock: effects of tetrodotoxin and high $\mathrm{Mg}^{21}$ Brain Res 573:336-340.

Prosser RA, Dean RR, Edgar DM, Heller HC, Miller JD (1993) Serotonin and the mammalian circadian system. I. In vitro phasc shifts by scrotonergic agonists and antagonists. J Biol Rhythms 8:1-16.

Prosser RA, Heller HC, Miller JD (1994a) Serotonergic phase advances of the mammalian circadian clock involve protein kinase $A$ and $K^{-}$ channel opening. Brain Res 644:67-73.

Prosser RA, Macdonald ES, Heller HC (1994b) c-fos mRNA in the suprachiasmatic nuclei in vitro shows a circadian rhythm and responds to a serotonergic agonist. Mol Brain Res 25:151-156.

Ramirez AD, Ramirez VD, Mcyer DC (1987) The nature and magnitude of in vivo 5-hydroxyindoleacetic acid output from 5-hydroxytryptamine terminals is related to specific regions of the suprachiasmatic nucleus. Neuroendocrinology 46:430-438.

Rea MA, Glass JD, Colwell CS (1994) Serotonin modulates the photic response in the hamster suprachiasmatic nuclei. J Neurosci 14:3635-3642.

Selim M, Glass JD, Hauser UE, Rea MA (1993) Serotonergic inhibition of light-induced fos protein expression and extracellular glutamate in the suprachiasmatic nuclei. Brain Res 621:181-188.
Shibata S, Moore RY (1988) Electrical and metabolic activity of suprachiasmatic nucleus neurons in hamster hypothalamic slices. Brain Res 438:374-378.

Sirn LJ, Juseph SA (1992) Efferent projections of the nucleus raphe magnus. Brain Res Bull 28:679-682.

Sim LJ, Joseph SA (1993) Dorsal raphe nucleus efferents: termination in peptidergic fields. Peptides 14:75-83.

Smale L, Michels KM, Moore RY, Morin LP (1990) Destruction of the hamster serotonergic system by 5,7-DHT: effects on circadian rhythm phase, entrainment and response to triazolam. Brain Res 515:9-19.

Steinbusch HWM (1981) Distribution of serotonin-immunoreactivity in the central nervous system of the rat-cell bodies and terminals. Neuroscience 6:557-618

Stcphan FK, Zucker I (1972) Circadian rhythms in drinking behavior and locomotor activity of rats are eliminated by hypothalamic lesions. Proc Natl Acad Sci USA 69:1583-1586.

Sutin EL, Jacobowitz DM (1988) Immunocytochemical localization of peptides and other neurochemicals in the rat laterodorsal tegmental nucleus and adjacent area. J Comp Ncurol 270:243-270.

Takatsuji K, Miguel-Hidalgo J-J, Tohyama M (1991) Retinal fibers make synaptic contact with neuropeptide $\mathrm{Y}$ and enkephalin immunoreactive neurons in the intergeniculate leaflet of the rat. Neurosei Lett 125:73-76.

Tominaga K, Shibata S, Ueki S, Watanabe S (1992) Effects of 5-HT receptor agonists on the circadian rhythm of whecl-running activity in hamsters. Eur I Pharmacol 214:79-84.

Tork I (1990) Anatomy of the scrotonergic system. Ann NY Acad Sci 6)(0):9-35.

Turek FW (1989) Effects of stimulated physical activity on the circadian pacemaker of vertebrates. J Biol Rhythms 4:135-147.

van de Kar LD, Lorens SA (1979) Differential scrotonergic innervation of individual hypothalamic nuclei and other forcbrain regions by the dorsal and median midbrain raphe nuclei. Brain Res 162:45-54.

Van Recth O, Turek FW (1989) Stimulated activity mediates phase shifts in the hamster circadian clock induced by dark pulses or benzodiazepines. Nature 339:49-51.

Van Reeth O, Zhang Y, Reddy A, Zee P, Turek FW (1993) Aging alters the entraining effects of an activity-inducing stimulus on the circadian clock. Brain Res 607:286-292.

Vaudano E, Legg CR (1992) Cerebellar connections of the ventral lateral geniculate nucleus in the rat. Anat Embryol 186:583-588.

Vertes RP (1984) A lectin horseradish peroxidase study of the origin of ascending fibers in the medial forcbrain bundle of the rat. The lower brainstem. Neuroscience 11:651-668.

Vertes RP (1991) A PHA-L analysis of ascending projections of the dorsal raphe nucleus in the rat. J Comp Neurol 313:643-668.

Vertes RP, Martin GF (1988) Autoradiographic analysis of ascending projections from the pontine and mesencephalic reticular formation and the median raphe in the rat. J Comp Neurol 275:511-541.

Villar MJ, Vitale ML, Hokfelt T, Verhofstad AAJ (1988) Dorsal raphe serotoninergic branching neurons projecting both to the lateral geniculate body and superior colliculus: a combined retrograde tracingimmunohistochemical in the rat. J Comp Ncurol 277:126-140.

Wallace JA, Lauder JM (1983) Development of the serotonergic system in the rat embryo: an immunocytochemical study. Brain Res Bull 10:459-479.

Waterhouse BD, Border B, Wahl L, Mihailoff GA (1993) Topographic organization of rat locus coeruleus and dorsal raphe nuclei: distribution of cells projecting to visual system structures. J Comp Neurol 336:345-361.

Wickland CR, Turek FW (1991) Phasc-shifting effects of acutc increases in activity on circadian locomotor rhythms in hamsters. Am J Physiol 261:R1109-R1117.

Wickland C, Turek FW (1994) Lesions of the thalamic intergeniculate leaflet block activity-induced phase shifts in the circadian activity rhythm of the golden hamster. Brain Res 660:293-300.

Ying S-W, Rusak B (1994) Effects of serotonergic agonists on firing rates of photically responsive cells in the hamster suprachiasmatic nucleus. Brain Res 651:37-46.

Ying S-W, Zhang D-X, Rusak B (1993) Effects of serotonin agonists and melatonin on photic responses of hamster intergeniculate leaflet ncurons. Brain Res 628:8-16.

Zhang DX, Rusak B (1989) Photic sensitivity of geniculate neurons that project to the suprachiasmatic nuclei or the contralateral geniculate. Brain Res 504:161-164. 